\title{
A Domain Decomposition Algorithm for Contact Problems: Analysis and Implementation
}

\author{
J. Haslinger ${ }^{a}$, R. Kučera ${ }^{b 1}$ and T. Sassi ${ }^{c}$ \\ ${ }^{a}$ Department of Numerical Mathematics, Charles University Prague, 18675 Prague, CZ \\ ${ }^{b}$ Department of Mathematics and Descriptive Geometry, VŠB-TU Ostrava, 70833 Ostrava, CZ \\ ${ }^{c}$ Department of Mathematics, University of Basse- Normandie, 14032 Caen, France
}

\begin{abstract}
The paper deals with an iterative method for numerical solving frictionless contact problems for two elastic bodies. Each iterative step consists of a Dirichlet problem for the one body, a contact problem for the other one and two Neumann problems to coordinate contact stresses. Convergence is proved by the Banach fixed point theorem in both continuous and discrete case. Numerical experiments indicate scalability of the algorithm for some choices of the relaxation parameter.
\end{abstract}

Key words: contact problem, domain decomposition method AMS subject classification: 74M15, 65Y05

\section{Introduction}

Contact problems take an important place in the computational mechanics (see [8], [10], [5], [1], [13], [14] and references therein). Many numerical procedures have been proposed in engineering literature. The discretization of such problems leads to very large and ill-conditioned systems. Domain decomposition methods represent a possible remedy how to overcome this difficulty. Recently, a Neumann-Dirichlet algorithm for solving frictionless Signorini contact problems between two elastic bodies has been proposed and studied in the continuous and discrete setting [15], [7],

\footnotetext{
${ }^{1}$ Corresponding author. E-mail: radek.kucera@vsb.cz
} 
[2]. Bayada, Sabil and Sassi proposed another approach in [3] and proved its convergence in the continuous setting. Each iterative step of their algorithm is given by a linear elasticity problem with prescribed displacements for one body and a contact problem with a prescribed gap function for the second one. Then two Neumann problems are used to ensure continuity of contact stresses along a common part of the boundary.

The purpose of this contribution is to present a modified version of the previous algorithm and to compare the resulting iterative schemes. This method is an extension of the one analyzed in [16] to variational inequalities.

The paper is organized as follows. Firstly in Section 2, we introduce the variational formulation of frictionless Signorini problems. In Section 3 we propose a modified version of the algorithm from [3] (see also [12]). Convergence in the continuous and discrete setting is established in next two sections. Finally, in Section 6 we present implementations of the modified and the original algorithm both based on dual formulations. Results of model examples will be shown in Section 7.

\section{Formulation of the Problem}

Let us consider two elastic bodies occupying bounded domains $\Omega^{\alpha} \in \mathbb{R}^{2}, \alpha=1,2$ with sufficiently smooth boundaries, see Fig. 1. The boundary $\Gamma^{\alpha}$ of $\Omega^{\alpha}$ consists of three non-empty disjoint parts $\Gamma_{u}^{\alpha}, \Gamma_{p}^{\alpha}, \Gamma_{c}^{\alpha}$ so that $\Gamma^{\alpha}=\bar{\Gamma}_{u}^{\alpha} \cup \bar{\Gamma}_{p}^{\alpha} \cup \bar{\Gamma}_{c}^{\alpha}$. The zero displacements will be prescribed on $\Gamma_{u}^{\alpha}$ and surface tractions of density $p^{\alpha} \in\left(L^{2}\left(\Gamma_{p}^{\alpha}\right)\right)^{2}$ act on $\Gamma_{p}^{\alpha}$. On the contact interface represented by $\Gamma_{c}^{1}$ and $\Gamma_{c}^{2}$,we consider the contact conditions: the non-penetration of the bodies and the transmission of forces. Friction will be neglected. The contact conditions are prescribed by a predefined unit critical vector $\nu \in\left(L^{2}\left(\Gamma_{c}^{1}\right)\right)^{2}$ (e.g. the outward unit normal vector to $\left.\Gamma_{c}^{1}\right)$. The initial gap between the bodies in the direction of $\nu$ is given by a function $g \in L^{2}\left(\Gamma_{c}^{1}\right)$. Let $\chi: \Gamma_{c}^{1} \rightarrow \Gamma_{c}^{2}, \chi(x)=$ $x+g(x) \nu(x), x \in \Gamma_{c}^{1}$, be a one-to-one transfer mapping so that we can define $\nu^{1}:=\nu$ on $\Gamma_{c}^{1}$ and $\nu^{2}:=\nu \circ \chi^{-1}$ on $\Gamma_{c}^{2}$. Finally, we suppose that the bodies $\Omega^{\alpha}$ are subject to volume forces of density $f^{\alpha} \in\left(L^{2}\left(\Omega^{\alpha}\right)\right)^{2}, \alpha=1,2$.

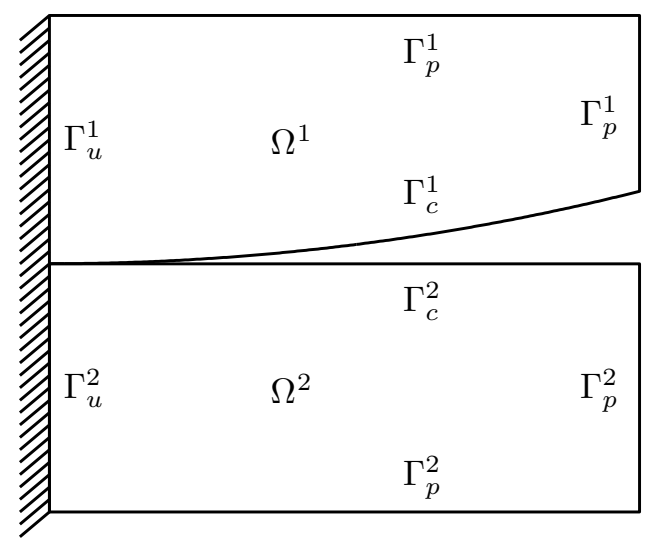

Figure 1: Geometry of the contact problem. 
Before giving the weak formulation of the contact problem, we introduce notation. Let us define the following spaces $(\alpha=1,2)$ :

$$
\begin{aligned}
V^{\alpha} & =\left\{v^{\alpha} \in H^{1}\left(\Omega^{\alpha}\right) \mid v^{\alpha}=0 \text { on } \Gamma_{u}^{\alpha}\right\}, \\
\mathbb{V}^{\alpha} & =V^{\alpha} \times V^{\alpha}, \\
\mathbb{V}_{0}^{\alpha} & =\left\{v^{\alpha} \in \mathbb{V}^{\alpha} \mid v^{\alpha} \cdot \nu^{\alpha}=0 \text { on } \Gamma_{c}^{\alpha}\right\},
\end{aligned}
$$

and

$$
\mathbb{V}=\mathbb{V}^{1} \times \mathbb{V}^{2}, \quad \mathbb{V}_{0}=\mathbb{V}_{0}^{1} \times \mathbb{V}_{0}^{2}
$$

The standard norm in $V^{\alpha}, \mathbb{V}^{\alpha}$ will be denoted by $\|\cdot\|_{1, \Omega^{\alpha}}$ in what follows. The bilinear form $a: \mathbb{V} \times \mathbb{V} \mapsto \mathbb{R}$ representing the inner energy of the system is given by:

$$
a(v, w)=a^{1}\left(v^{1}, w^{1}\right)+a^{2}\left(v^{2}, w^{2}\right), \quad v, w \in \mathbb{V},
$$

with

$$
a^{\alpha}\left(v^{\alpha}, w^{\alpha}\right)=\int_{\Omega^{\alpha}} a_{i j k l}^{\alpha} e_{i j}\left(v^{\alpha}\right) e_{k l}\left(w^{\alpha}\right) d x, \quad \alpha=1,2,
$$

where $a_{i j k l}^{\alpha} \in L^{\infty}\left(\Omega^{\alpha}\right)$ are coefficients of a symmetric, positive definite tensor of the fourth order defining the linear Hook law, $v=\left(v^{1}, v^{2}\right), w=\left(w^{1}, w^{2}\right)$ and $e_{i j}\left(v^{\alpha}\right)=\left(\partial_{i} v_{j}^{\alpha}+\partial_{j} v_{i}^{\alpha}\right) / 2, i, j, k, l=$ 1,2 . By $b: \mathbb{V} \mapsto \mathbb{R}$ we denote the work of applied forces:

$$
b(v)=b^{1}\left(v^{1}\right)+b^{2}\left(v^{2}\right), \quad v \in \mathbb{V}
$$

where

$$
b^{\alpha}\left(v^{\alpha}\right)=\int_{\Omega^{\alpha}} f^{\alpha} \cdot v^{\alpha} d x+\int_{\Gamma_{p}^{\alpha}} p^{\alpha} \cdot v^{\alpha} d s, \quad \alpha=1,2 .
$$

The weak formulation of the contact problem reads as follows:

$$
\left.\begin{array}{l}
\text { Find } u \in \mathbb{K} \text { such that } \\
a(u, v-u) \geq b(v-u) \quad \forall v \in \mathbb{K},
\end{array}\right\}
$$

where $\mathbb{K}$ is the convex set of kinematically admissible displacements,

$$
\mathbb{K}=\left\{v \in \mathbb{V} \mid v_{\nu}^{1}-v_{\nu}^{2} \leq g \text { on } \Gamma_{c}^{1}\right\}
$$

with $v_{\nu}^{1}:=v^{1} \cdot \nu^{1}$ and $v_{\nu}^{2}:=\left(v^{2} \circ \chi\right) \cdot\left(\nu^{2} \circ \chi\right)$. It is well-known that $(\mathcal{P})$ has a unique solution [13, $11]$.

The symbol $\sigma\left(v^{\alpha}\right), v^{\alpha} \in \mathbb{V}^{\alpha}, \alpha=1,2$ stands for the stress field in $\Omega^{\alpha}$ :

$$
\sigma_{i j}\left(v^{\alpha}\right)=a_{i j k l}^{\alpha} e_{k l}\left(v^{\alpha}\right), \quad i, j=1,2 .
$$

The normal and tangential components of $\sigma\left(v^{\alpha}\right)$ on $\Gamma_{c}^{\alpha}$ are given by:

$$
\sigma_{\nu}\left(v^{\alpha}\right):=\sigma_{i j}\left(v^{\alpha}\right) \nu_{i}^{\alpha} \nu_{j}^{\alpha}, \quad \sigma_{t}\left(v^{\alpha}\right):=\sigma_{i j}\left(v^{\alpha}\right) \nu_{j}^{\alpha}-\sigma_{\nu}\left(v^{\alpha}\right) \nu^{\alpha} .
$$




\section{Algorithm}

For the sake of simplicity of our presentation we shall suppose that there is no gap between $\Omega^{1}$ and $\Omega^{2}$ in the undeformed state. Then $\Gamma_{c}^{1}=\Gamma_{c}^{2}=: \Gamma_{c}, g=0$ and $\chi=\mathrm{id}$ on $\Gamma_{c}$. In what follows the symbol $\nu$ denotes the outward unit normal vector on $\Gamma_{c}$ with respect to $\Omega^{1}$, while $\nu^{\alpha}$ stands for the outward unit normal vector to $\Omega^{\alpha}$ on the remaining parts of $\Gamma^{\alpha}$.

Next we shall suppose that $\Gamma_{c}$ is of the class $C^{1,1}$ and $\bar{\Gamma}_{u}^{1} \cap \bar{\Gamma}_{c}=\bar{\Gamma}_{u}^{2} \cap \bar{\Gamma}_{c}$, i.e., either $\operatorname{dist}\left(\Gamma_{u}^{\alpha}, \Gamma_{c}\right)>$ $0, \alpha=1,2$ or $\bar{\Gamma}_{u}^{1}, \bar{\Gamma}_{u}^{2}$ meet $\bar{\Gamma}_{c}$ at the same points. Let

$$
\begin{aligned}
& H^{1 / 2}\left(\Gamma_{c}\right)=\left\{\varphi \in L^{2}\left(\Gamma_{c}\right) \mid \exists v^{\alpha} \in V^{\alpha}, v^{\alpha}=\varphi \text { on } \Gamma_{c}\right\}, \\
& H^{-1 / 2}\left(\Gamma_{c}\right)=\left(H^{1 / 2}\left(\Gamma_{c}\right)\right)^{\prime} \quad\left(\text { dual of } H^{1 / 2}\left(\Gamma_{c}\right)\right), \\
& \mathbb{H}^{1 / 2}\left(\Gamma_{c}\right)=H^{1 / 2}\left(\Gamma_{c}\right) \times H^{1 / 2}\left(\Gamma_{c}\right) .
\end{aligned}
$$

In view of the assumption on the mutual position of $\Gamma_{u}^{\alpha}$ and $\Gamma_{c}$ it is easy to see that the definition of $H^{1 / 2}\left(\Gamma_{c}\right)$ does not depend on the choice of $V^{\alpha}, \alpha=1$, 2. It is also well-known that $H^{1 / 2}\left(\Gamma_{c}\right)$ is the Banach space with the norm

$$
\|\varphi\|_{\frac{1}{2}}^{2}:=\int_{\Gamma_{c}} \varphi^{2} d s+\int_{\Gamma_{c}} \int_{\Gamma_{c}} \frac{|\varphi(x)-\varphi(y)|^{2}}{|x-y|^{2}} d s_{x} d s_{y} .
$$

In addition, if $v^{\alpha} \in \mathbb{V}^{\alpha}$ then $v_{\nu}^{\alpha} \in H^{1 / 2}\left(\Gamma_{c}\right)$ as follows from the smoothness assumption on $\Gamma_{c}$. The duality pairing between $H^{-1 / 2}\left(\Gamma_{c}\right)$ and $H^{1 / 2}\left(\Gamma_{c}\right)$ will be denoted by $\langle$,$\rangle in what follows.$

In order to split problem $(\mathcal{P})$ into two subproblems coupled through the contact interface, we introduce the mapping that builts functions in $\Omega^{\alpha}$ from their values on $\Gamma_{c}$. Let $P_{\alpha}: H^{1 / 2}\left(\Gamma_{c}\right) \mapsto \mathbb{V}^{\alpha}$ be the extension operator defined by $P_{\alpha} \varphi=v^{\alpha}, \varphi \in H^{1 / 2}\left(\Gamma_{c}\right)$, where $v^{\alpha} \in \mathbb{V}^{\alpha}, \alpha=1,2$ satisfies:

$$
\left.\begin{array}{rl}
a^{\alpha}\left(v^{\alpha}, z^{\alpha}\right)=0 & \forall z^{\alpha} \in \mathbb{V}_{0}^{\alpha}, \\
v_{\nu}^{\alpha}=\varphi & \text { on } \Gamma_{c},
\end{array}\right\}
$$

or, equivalently,

$$
\left.\begin{array}{rl}
\operatorname{div} \sigma\left(v^{\alpha}\right)=0 & \text { in } \Omega^{\alpha}, \\
v^{\alpha}=0 & \text { on } \Gamma_{u}^{\alpha}, \\
\sigma\left(v^{\alpha}\right) \cdot \nu^{\alpha}=0 & \text { on } \Gamma_{p}^{\alpha}, \\
\sigma_{t}\left(v^{\alpha}\right)=0 & \text { on } \Gamma_{c}, \\
v_{\nu}^{\alpha}=\varphi & \text { on } \Gamma_{c} .
\end{array}\right\}
$$

With any $\varphi \in H^{1 / 2}\left(\Gamma_{c}\right)$ we associate the closed convex set

$$
\mathbb{K}^{2}(\varphi)=\left\{v^{2} \in \mathbb{V}^{2} \mid \varphi-v_{\nu}^{2} \leq 0 \text { on } \Gamma_{c}\right\} .
$$

Our algorithm is motivated by the following observation. Let $\lambda \in H^{1 / 2}\left(\Gamma_{c}\right)$ be given and let $u^{1}, u^{2}$ be solutions of:

$$
\left.\begin{array}{l}
\text { Find } u^{1}:=u^{1}(\lambda) \in \mathbb{V}^{1} \text { such that } \\
a^{1}\left(u^{1}, v^{1}\right)=b^{1}\left(v^{1}\right) \quad \forall v^{1} \in \mathbb{V}_{0}^{1}, \\
u_{\nu}^{1}=\lambda \text { on } \Gamma_{c},
\end{array}\right\}
$$


and

$$
\left.\begin{array}{l}
\text { Find } u^{2}:=u^{2}(\lambda) \in \mathbb{K}^{2}(\lambda) \text { such that } \\
a^{2}\left(u^{2}, v^{2}-u^{2}\right) \geq b^{2}\left(v^{2}-u^{2}\right) \quad \forall v^{2} \in \mathbb{K}^{2}(\lambda) .
\end{array}\right\}
$$

Then $u=\left(u^{1}, u^{2}\right)$ solves the original contact problem if and only if the contact stresses are continuously transmitted through $\Gamma_{c}$ (the law of action and reaction). Since $\sigma_{t}\left(u^{1}\right)=\sigma_{t}\left(u^{2}\right)=0$ on $\Gamma_{c}$ as follows from (3.4) and (3.5), one needs only to guarantee that

$$
\sigma_{\nu}\left(u^{1}\right)=\sigma_{\nu}\left(u^{2}\right) \text { on } \Gamma_{c} .
$$

Suppose that $\lambda$ is chosen in such a way that $w_{\nu}^{1}=w_{\nu}^{2}$ on $\Gamma_{c}$, where $w^{1} \in \mathbb{V}^{1}, w^{2} \in \mathbb{V}^{2}$ solve the following problems:

$$
\begin{aligned}
& a^{1}\left(w^{1}, v^{1}\right)=\frac{1}{2}\left(-a^{1}\left(u^{1}, v^{1}\right)+b^{1}\left(v^{1}\right)-a^{2}\left(u^{2}, P_{2} v^{1}\right)+b^{2}\left(P_{2} v^{1}\right)\right) \quad \forall v^{1} \in \mathbb{V}^{1}, \\
& a^{2}\left(w^{2}, v^{2}\right)=\frac{1}{2}\left(a^{2}\left(u^{2}, v^{2}\right)-b^{2}\left(v^{2}\right)+a^{1}\left(u^{1}, P_{1} v^{2}\right)-b^{1}\left(P_{1} v^{2}\right)\right) \quad \forall v^{2} \in \mathbb{V}^{2} .
\end{aligned}
$$

If $w_{\nu}^{1}=w_{\nu}^{2}$ on $\Gamma_{c}$ then $a^{1}\left(w^{1}, w^{1}\right)+a^{2}\left(w^{2}, w^{2}\right)=0$. Therefore $w^{\alpha}=0$ in $\Omega^{\alpha}, \alpha=1,2$, implying (3.6).

We propose the following algorithm.

$\underline{\text { AlgORITHM } 3.1}$ Let $\lambda_{0} \in H^{1 / 2}\left(\Gamma_{c}\right)$ and $\theta>0$ be given. For $k \geq 1$ compute $u_{k}^{\alpha}, w_{k}^{\alpha}, \alpha=1,2$, and $\lambda_{k}$ as follows:

(Step 1)

$$
\left.\begin{array}{l}
\text { Find } u_{k}^{1} \in \mathbb{V}^{1} \text { such that } \\
a^{1}\left(u_{k}^{1}, v^{1}\right)=b^{1}\left(v^{1}\right) \quad \forall v^{1} \in \mathbb{V}_{0}^{1}, \\
u_{k, \nu}^{1}=\lambda_{k-1} \text { on } \Gamma_{c} .
\end{array}\right\}
$$

(Step 2)

$$
\left.\begin{array}{l}
\text { Find } u_{k}^{2} \in \mathbb{K}^{2}\left(\lambda_{k-1}\right) \text { such that } \\
a^{2}\left(u_{k}^{2}, v^{2}-u_{k}^{2}\right) \geq b^{2}\left(v^{2}-u_{k}^{2}\right) \quad \forall v^{2} \in \mathbb{K}^{2}\left(\lambda_{k-1}\right) .
\end{array}\right\}
$$

(Step 3)

$$
\left.\begin{array}{l}
\text { Find } w_{k}^{1} \in \mathbb{V}^{1} \text { such that } \\
a^{1}\left(w_{k}^{1}, v^{1}\right)=\frac{1}{2}\left(-a^{1}\left(u_{k}^{1}, v^{1}\right)+b^{1}\left(v^{1}\right)-a^{2}\left(u_{k}^{2}, P_{2} v^{1}\right)+b^{2}\left(P_{2} v^{1}\right)\right) \quad \forall v^{1} \in \mathbb{V}^{1} .
\end{array}\right\}
$$

(Step 4)

$$
\left.\begin{array}{l}
\text { Find } w_{k}^{2} \in \mathbb{V}^{2} \text { such that } \\
a^{2}\left(w_{k}^{2}, v^{2}\right)=\frac{1}{2}\left(a^{2}\left(u_{k}^{2}, v^{2}\right)-b^{2}\left(v^{2}\right)+a^{1}\left(u_{k}^{1}, P_{1} v^{2}\right)-b^{1}\left(P_{1} v^{2}\right)\right) \quad \forall v^{2} \in \mathbb{V}^{2} .
\end{array}\right\}
$$

(Step 5)

$$
\lambda_{k}=\lambda_{k-1}+\theta\left(w_{k, \nu}^{1}-w_{k, \nu}^{2}\right) .
$$


Remark 1. Let us note that our algorithm is the modification of the one analyzed in [2, 17], where Bayada, Sabil and Sassi used the extension operator $R_{\alpha}: \mathbb{H}^{1 / 2}\left(\Gamma_{c}\right) \mapsto \mathbb{V}^{\alpha}$ defined by $R_{\alpha} \varphi=v^{\alpha}, \varphi \in \mathbb{H}^{1 / 2}\left(\Gamma_{c}\right)$ with $v^{\alpha} \in \mathbb{V}^{\alpha}$ such that:

$$
\left.\begin{array}{ll}
a^{\alpha}\left(v^{\alpha}, w^{\alpha}\right)=0 & \forall w^{\alpha} \in \mathbb{V}^{\alpha}, \\
v^{\alpha}=\varphi & \text { on } \Gamma_{c} .
\end{array}\right\}
$$

This choice of the extension operator gives a procedure in which continuity not only of the normal but also of the tangential component of the contact stress has to be ensured.

\section{Convergence analysis}

The aim of this section is to prove convergence of ALGORITHM 3.1. We shall use the Banach fixed point theorem for a suitably defined operator. First we reformulate the steps of the previous algorithm in terms of operators acting on $\Gamma_{c}$.

Let $S_{\alpha}: H^{1 / 2}\left(\Gamma_{c}\right) \mapsto H^{-1 / 2}\left(\Gamma_{c}\right)$ be the following Poincaré-Steklov operator: for any $\varphi \in$ $H^{1 / 2}\left(\Gamma_{c}\right)$

$$
S_{\alpha} \varphi=\sigma_{\nu}\left(u^{\alpha}\right) \text { on } \Gamma_{c}, \alpha=1,2,
$$

where $u^{\alpha}$ solves

$$
\left.\begin{array}{rl}
\operatorname{div} \sigma\left(u^{\alpha}\right)=0 & \text { in } \Omega^{\alpha}, \\
u^{\alpha}=0 & \text { on } \Gamma_{u}^{\alpha}, \\
\sigma\left(u^{\alpha}\right) \cdot \nu^{\alpha}=0 & \text { on } \Gamma_{p}^{\alpha}, \\
\sigma_{t}\left(u^{\alpha}\right)=0 & \text { on } \Gamma_{c}, \\
u_{\nu}^{\alpha}=\varphi & \text { on } \Gamma_{c},
\end{array}\right\}
$$

i.e., $u^{\alpha}=P_{\alpha} \varphi, \alpha=1,2$. From this and (4.1) we obtain the equivalent definition of $S_{\alpha}$ :

$$
\left\langle S_{\alpha} \varphi, \psi\right\rangle=a^{\alpha}\left(P_{\alpha} \varphi, v^{\alpha}\right) \quad \forall v^{\alpha} \in \mathbb{V}^{\alpha}, \quad v_{\nu}^{\alpha}=(-1)^{\alpha-1} \psi \text { on } \Gamma_{c},
$$

where the sign of $\psi$ is due to the orientation of $\nu$ on $\Gamma_{c}$.

In order to decouple the influence of the applied and contact forces, we introduce $U^{1} \in \mathbb{V}_{0}^{1}$ as the solution of

$$
a^{1}\left(U^{1}, v^{1}\right)=b^{1}\left(v^{1}\right) \quad \forall v^{1} \in \mathbb{V}_{0}^{1},
$$

which is the weak formulation of

$$
\left.\begin{array}{ll}
-\operatorname{div} \sigma\left(U^{1}\right)=f^{1} & \text { in } \Omega^{1}, \\
U^{1}=0 & \text { on } \Gamma_{u}^{1}, \\
\sigma\left(U^{1}\right) \cdot \nu^{1}=p^{1} & \text { on } \Gamma_{p}^{1}, \\
\sigma_{t}\left(U^{1}\right)=0 & \text { on } \Gamma_{c}, \\
U_{\nu}^{1}=0 & \text { on } \Gamma_{c} .
\end{array}\right\}
$$


Thus $u_{k}^{1}$ solving (3.7) can be written as:

$$
u_{k}^{1}=U^{1}+P_{1} \lambda_{k-1} .
$$

The function $u_{k}^{2}$ from (3.8) solves the following unilateral problem :

$$
\left.\begin{array}{ll}
-\operatorname{div} \sigma\left(u_{k}^{2}\right)=f^{2} & \text { in } \Omega^{2}, \\
u_{k}^{2}=0 & \text { on } \Gamma_{u}^{2}, \\
\sigma\left(u_{k}^{2}\right) \cdot \nu^{2}=p^{2} & \text { on } \Gamma_{p}^{2}, \\
\sigma_{\nu}\left(u_{k}^{2}\right) \leq 0 & \text { on } \Gamma_{c}, \\
\lambda_{k-1}-u_{k, \nu}^{2} \leq 0 & \text { on } \Gamma_{c}, \\
\sigma_{\nu}\left(u_{k}^{2}\right)\left(\lambda_{k-1}-u_{k, \nu}^{2}\right)=0 & \text { on } \Gamma_{c}, \\
\sigma_{t}\left(u_{k}^{2}\right)=0 & \text { on } \Gamma_{c} .
\end{array}\right\}
$$

As before, we decouple $u_{k}^{2}$. Let $U^{2}$ be the solution of:

$$
\left.\begin{array}{ll}
-\operatorname{div} \sigma\left(U^{2}\right)=f^{2} & \text { in } \Omega^{2}, \\
U^{2}=0 & \text { on } \Gamma_{u}^{2}, \\
\sigma\left(U^{2}\right) \cdot \nu^{2}=p^{2} & \text { on } \Gamma_{p}^{2}, \\
\sigma_{\nu}\left(U^{2}\right)=0 & \text { on } \Gamma_{c}, \\
\sigma_{t}\left(U^{2}\right)=0 & \text { on } \Gamma_{c} .
\end{array}\right\}
$$

Then

$$
u_{k}^{2}=U^{2}+\widetilde{u}_{k}^{2}
$$

where $\widetilde{u}_{k}^{2}$ solves:

$$
\begin{array}{ll}
\operatorname{div} \sigma\left(\widetilde{u}_{k}^{2}\right)=0 & \text { in } \Omega^{2}, \\
\widetilde{u}_{k}^{2}=0 & \text { on } \Gamma_{u}^{2} \\
\sigma\left(\widetilde{u}_{k}^{2}\right) \cdot \nu^{2}=0 & \text { on } \Gamma_{p}^{2} \\
\sigma_{\nu}\left(\widetilde{u}_{k}^{2}\right) \leq 0 & \text { on } \Gamma_{c} \\
\lambda_{k-1}-U_{\nu}^{2}-\widetilde{u}_{k, \nu}^{2} \leq 0 & \text { on } \Gamma_{c} \\
\sigma_{\nu}\left(\widetilde{u}_{k}^{2}\right)\left(\lambda_{k-1}-U_{\nu}^{2}-\widetilde{u}_{k, \nu}^{2}\right)=0 & \text { on } \Gamma_{c} \\
\sigma_{t}\left(\widetilde{u}_{k}^{2}\right)=0 & \text { on } \Gamma_{c} .
\end{array}
$$

The weak formulation of (4.9) is given by:

$$
\left.\begin{array}{l}
\text { Find } \widetilde{u}_{k}^{2} \in \mathbb{K}^{2}\left(\lambda_{k-1}-U_{\nu}^{2}\right) \text { such that } \\
a^{2}\left(\widetilde{u}_{k}^{2}, v^{2}-\widetilde{u}_{k}^{2}\right) \geq 0 \quad \forall v^{2} \in \mathbb{K}^{2}\left(\lambda_{k-1}-U_{\nu}^{2}\right) .
\end{array}\right\}
$$


Let $Q_{2}: H^{1 / 2}\left(\Gamma_{c}\right) \mapsto H^{1 / 2}\left(\Gamma_{c}\right)$ be defined by:

$$
Q_{2} \lambda=v_{\nu}^{2} \quad \forall \lambda \in H^{1 / 2}\left(\Gamma_{c}\right),
$$

where $v^{2} \in K^{2}\left(\lambda-U_{\nu}^{2}\right)$ solves

$$
a^{2}\left(v^{2}, z^{2}-v^{2}\right) \geq 0 \quad \forall z^{2} \in K^{2}\left(\lambda-U_{\nu}^{2}\right) .
$$

Then $\widetilde{u}_{k, \nu}^{2}=Q_{2} \lambda_{k-1}$ and

$$
u_{k}^{2}=U^{2}+P_{2} Q_{2} \lambda_{k-1} .
$$

The solutions to (3.9) and (3.10) can be written by means of the Green formula and the Poincaré-Steklov operators $S_{1}$ and $S_{2}$ as follows:

$$
\begin{aligned}
\left\langle S_{1} w_{k, \nu}^{1}, v_{\nu}^{1}\right\rangle & =a^{1}\left(w_{k}^{1}, v^{1}\right)=\frac{1}{2}\left\langle\sigma_{\nu}\left(u_{k}^{2}\right)-\sigma_{\nu}\left(u_{k}^{1}\right), v_{\nu}^{1}\right\rangle, \\
-\left\langle S_{2} w_{k, \nu}^{2}, v_{\nu}^{2}\right\rangle & =a^{2}\left(w_{k}^{2}, v^{2}\right)=\frac{1}{2}\left\langle-\sigma_{\nu}\left(u_{k}^{2}\right)+\sigma_{\nu}\left(u_{k}^{1}\right), v_{\nu}^{2}\right\rangle .
\end{aligned}
$$

Therefore

$$
\begin{aligned}
& w_{k, \nu}^{1}=\frac{1}{2} S_{1}^{-1}\left(\sigma_{\nu}\left(u_{k}^{2}\right)-\sigma_{\nu}\left(u_{k}^{1}\right)\right), \\
& w_{k, \nu}^{2}=\frac{1}{2} S_{2}^{-1}\left(\sigma_{\nu}\left(u_{k}^{2}\right)-\sigma_{\nu}\left(u_{k}^{1}\right)\right) .
\end{aligned}
$$

Using these representations we can express Step 5. of the algorithm as follows:

$$
\begin{aligned}
\lambda_{k} & =\lambda_{k-1}-\frac{\theta}{2}\left(S_{1}^{-1}-S_{2}^{-1}\right)\left(\sigma_{\nu}\left(u_{k}^{1}\right)-\sigma_{\nu}\left(u_{k}^{2}\right)\right) \\
& =\lambda_{k-1}-\frac{\theta}{2} M\left(S_{1} u_{k, \nu}^{1}-S_{2} u_{k, \nu}^{2}\right),
\end{aligned}
$$

where $M:=S_{1}^{-1}-S_{2}^{-1}$. Since $u_{k, \nu}^{1}=\lambda_{k-1}$ and $u_{k, \nu}^{2}=U_{\nu}^{2}+Q_{2} \lambda_{k-1}$, it follows that

$$
\lambda_{k}=\lambda_{k-1}-\frac{\theta}{2} M\left(S_{1} \lambda_{k-1}-S_{2} U_{\nu}^{2}-S_{2} Q_{2} \lambda_{k-1}\right) .
$$

To simplify notation, let $T_{\theta}: H^{1 / 2}\left(\Gamma_{c}\right) \mapsto H^{1 / 2}\left(\Gamma_{c}\right)$ be defined by:

$$
T_{\theta} \lambda:=\lambda-\frac{\theta}{2} M\left(S_{1} \lambda-S_{2} Q_{2} \lambda+g_{0}\right), \quad \lambda \in H^{1 / 2}\left(\Gamma_{c}\right),
$$

where $g_{0}=-S_{2} U_{\nu}^{2}$. Then (4.12) is equivalent to

$$
\lambda_{k}=T_{\theta} \lambda_{k-1}
$$

From the properties of $S_{\alpha}^{-1}, \alpha=1,2$ formulated in the next lemma it follows that $M$ is a one-toone mapping. Thus if $\bar{\lambda} \in H^{1 / 2}\left(\Gamma_{c}\right)$ is a fixed point of $T_{\theta}$ then $\bar{\lambda}$ is the searched $u_{\nu}^{1}$ on $\Gamma_{c}$ which guarantees the satisfaction of (3.6).

To prove the existence (and uniqueness) of the fixed point and convergence of the iterative scheme (4.13), we shall need the following results. 
Lemma 2. (i) The mappings $S_{\alpha}: H^{1 / 2}\left(\Gamma_{c}\right) \mapsto H^{-1 / 2}\left(\Gamma_{c}\right), \alpha=1,2$ are bounded, bijective, self-adjoint and the bilinear forms generated by $S_{\alpha}, S_{\alpha}^{-1}$ are elliptic in the corresponding spaces:

$$
\begin{aligned}
& \exists \bar{c}_{\alpha}>0:(-1)^{\alpha-1}\left\langle S_{\alpha} \varphi, \varphi\right\rangle \geq \bar{c}_{\alpha}\|\varphi\|_{\frac{1}{2}}^{2} \quad \forall \varphi \in H^{1 / 2}\left(\Gamma_{c}\right), \\
& \exists \bar{d}_{\alpha}>0:(-1)^{\alpha-1}\left\langle\mu, S_{\alpha}^{-1} \mu\right\rangle \geq \bar{d}_{\alpha}\|\mu\|_{-\frac{1}{2}}^{2} \quad \forall \mu \in H^{-1 / 2}\left(\Gamma_{c}\right) .
\end{aligned}
$$

(ii) The mapping $S_{2} Q_{2}: H^{1 / 2}\left(\Gamma_{c}\right) \mapsto H^{-1 / 2}\left(\Gamma_{c}\right)$ is Lipschitz continuous and monotone:

$$
\begin{aligned}
& \exists \bar{L}>0:\left\|S_{2} Q_{2} \psi-S_{2} Q_{2} \varphi\right\|_{-\frac{1}{2}} \leq \bar{L}\|\psi-\varphi\|_{\frac{1}{2}} \forall \psi, \varphi \in H^{1 / 2}\left(\Gamma_{c}\right), \\
& \left\langle S_{2} Q_{2} \psi-S_{2} Q_{2} \varphi, \psi-\varphi\right\rangle \leq 0 \quad \forall \psi, \varphi \in H^{1 / 2}\left(\Gamma_{c}\right) .
\end{aligned}
$$

Proof. $(i)$ are the standard properties of the Poincaré-Steklov operator. For the proof of $(i i)$ we refer to [7] and also to the next section, where it will be done in the discrete case.

To apply the Banach fixed point theorem, the space $H^{1 / 2}\left(\Gamma_{c}\right)$ will be equipped with the scalar product $\langle\psi, \varphi\rangle_{M^{-1}}:=\left\langle M^{-1} \psi, \varphi\right\rangle$ and the norm $\|\varphi\|_{M^{-1}}:=\langle\varphi, \varphi\rangle_{M^{-1}}^{1 / 2}$. From the definition of $M$ and (4.14), (4.15) it follows that $\|\cdot\|_{M^{-1}}$ is an equivalent norm to $\|\cdot\|_{\frac{1}{2}}$ in $H^{1 / 2}\left(\Gamma_{c}\right)$. Using this new norm, (4.14) and (4.16) read as follows:

$$
\begin{aligned}
& \exists c_{\alpha}>0:(-1)^{\alpha-1}\left\langle S_{\alpha} \varphi, \varphi\right\rangle \geq c_{\alpha}\|\varphi\|_{M^{-1}}^{2} \quad \forall \varphi \in H^{1 / 2}\left(\Gamma_{c}\right), \\
& \exists L>0:\left\|S_{2} Q_{2} \psi-S_{2} Q_{2} \varphi\right\|_{-\frac{1}{2}} \leq L\|\psi-\varphi\|_{M^{-1}} \quad \forall \psi, \varphi \in H^{1 / 2}\left(\Gamma_{c}\right) .
\end{aligned}
$$

Finally, the norm of a linear mapping $\mathcal{F}$ into $H^{1 / 2}\left(\Gamma_{c}\right)$ will be denoted by $\|\mathcal{F}\|$ and $\|\mid \mathcal{F}\|$ if $H^{1 / 2}\left(\Gamma_{c}\right)$ is equipped with the norm $\|\cdot\|_{\frac{1}{2}}$ and $\|\cdot\|_{M^{-1}}$, respectively.

Theorem 3. There exists $\theta_{0}>0$ such that the mapping $T_{\theta}$ is contractive for any $\theta \in\left(0, \theta_{0}\right)$.

Proof. From the definition of $T_{\theta}$ we have:

$$
\begin{aligned}
\left\|T_{\theta} \psi-T_{\theta} \varphi\right\|_{M^{-1}}^{2}= & \|\psi-\varphi\|_{M^{-1}}^{2}+\frac{\theta^{2}}{4}\left\|M S_{1}(\psi-\varphi)\right\|_{M^{-1}}^{2}+\frac{\theta^{2}}{4}\left\|M S_{2} Q_{2} \psi-M S_{2} Q_{2} \varphi\right\|_{M^{-1}}^{2} \\
& -\theta\left\langle M S_{1}(\psi-\varphi), \psi-\varphi\right\rangle_{M^{-1}}+\theta\left\langle M S_{2} Q_{2} \psi-M S_{2} Q_{2} \varphi, \psi-\varphi\right\rangle_{M^{-1}} \\
& -\frac{\theta^{2}}{2}\left\langle M S_{2} Q_{2} \psi-M S_{2} Q_{2} \varphi, M S_{1}(\psi-\varphi)\right\rangle_{M^{-1}}
\end{aligned}
$$

We shall estimate each term on the right hand-side of (4.20). It holds:

$$
\left\|M S_{1}(\psi-\varphi)\right\|_{M^{-1}}^{2} \leq\left\|M S_{1}\right\|^{2}\|\psi-\varphi\|_{M^{-1}}^{2}
$$


From (4.19) we obtain:

$$
\begin{aligned}
\left\|M S_{2} Q_{2} \psi-M S_{2} Q_{2}\right\|_{M^{-1}}^{2} & =\left\langle S_{2} Q_{2} \psi-S_{2} Q_{2} \varphi, M S_{2} Q_{2} \psi-M S_{2} Q_{2} \varphi\right\rangle \\
& \leq\left\|S_{2} Q_{2} \psi-S_{2} Q_{2} \varphi\right\|_{-\frac{1}{2}}\left\|M\left(S_{2} Q_{2} \psi-S_{2} Q_{2} \varphi\right)\right\|_{\frac{1}{2}} \\
& \leq\|M\| L^{2}\|\psi-\varphi\|_{M^{-1}}^{2} .
\end{aligned}
$$

Further, (4.18) yields:

$$
\left\langle M S_{1}(\psi-\varphi), \psi-\varphi\right\rangle_{M^{-1}}=\left\langle S_{1}(\psi-\varphi), \psi-\varphi\right\rangle \geq c_{1}\|\psi-\varphi\|_{M^{-1}}^{2}
$$

The last but one term in (4.20) will be neglected since

$$
\left\langle M S_{2} Q_{2} \psi-M S_{2} Q_{2} \varphi, \psi-\varphi\right\rangle_{M^{-1}}=\left\langle S_{2} Q_{2} \psi-S_{2} Q_{2} \varphi, \psi-\varphi\right\rangle \leq 0
$$

as follows from (4.17). Finally,

$$
\begin{aligned}
\left|\left\langle M S_{2} Q_{2} \psi-M S_{2} Q_{2} \varphi, M S_{1}(\psi-\varphi)\right\rangle_{M^{-1}}\right| & =\left|\left\langle S_{2} Q_{2} \psi-S_{2} Q_{2} \varphi, M S_{1}(\psi-\varphi)\right\rangle\right| \\
& \leq L\left\||| M S_{1} \mid\right\| \cdot\|\psi-\varphi\|_{M^{-1}}^{2}
\end{aligned}
$$

From (4.21)-(4.25) we see that

$$
\left\|T_{\theta} \psi-T_{\theta} \varphi\right\|_{M^{-1}}^{2} \leq\left(1+\frac{\theta^{2}}{4}\left|\left\|M S_{1}\right\|\right|^{2}+\frac{\theta^{2}}{4}\|M\| L^{2}+\frac{\theta^{2}}{2} L\left|\left\|M S_{1}\right\|\right|-\theta c_{1}\right)\|\psi-\varphi\|_{M^{-1}}^{2} .
$$

Thus $\theta_{0}=4 c_{1} /\left(||\left|M S_{1}\right|\left\|^{2}+\right\| M\left\|L^{2}+2 L\left|\left\|M S_{1} \mid\right\|\right)\right.\right.$ has the required property.

\section{Convergence in Discrete Setting}

In this section we shall suppose that both $\Omega^{1}, \Omega^{2}$ are polygonal domains with triangulations $\mathcal{T}_{h}^{\alpha}$ which are consistent with the decomposition of the boundary $\Gamma^{\alpha}$ into $\Gamma_{u}^{\alpha}, \Gamma_{p}^{\alpha}$ and $\Gamma_{c}, \alpha=1,2$. Moreover, let $\Gamma_{c}$ be represented by a straight segment. We restrict ourselves to the geometrical conforming situation with matching grids on $\Gamma_{c}$. On $\Omega^{\alpha}, \alpha=1,2$ we construct the finite element space $V_{h}^{\alpha}$ of piecewise linear functions:

$$
V_{h}^{\alpha}=\left\{v^{\alpha} \in C\left(\bar{\Omega}^{\alpha}\right) \mid v_{\mid T}^{\alpha} \in P^{1}(T) \text { for all } T \in \mathcal{T}_{h}^{\alpha}\right\} \cap V^{\alpha} .
$$

The restriction of $V_{h}^{\alpha}$ on the interface $\Gamma_{c}$ is denoted by $W_{h}^{\alpha}$ :

$$
W_{h}^{\alpha}=V_{\left.h\right|_{\Gamma_{c}}}^{\alpha} .
$$

In view of our assumptions on $\mathcal{T}_{h}^{\alpha}$ and the decomposition of $\Gamma^{\alpha}, W_{h}^{1}=W_{h}^{2}=: W_{h}$. Finally, let $\mathbb{V}_{h}^{\alpha}=V_{h}^{\alpha} \times V_{h}^{\alpha}, \alpha=1,2$, and $\mathbb{V}_{h}=\mathbb{V}_{h}^{1} \times \mathbb{V}_{h}^{2}$. By $\mathbb{V}_{0, h}^{\alpha}$ we denote the finite element subspace of $\mathbb{V}_{0}^{\alpha}:$

$$
\mathbb{V}_{0, h}^{\alpha}=\left\{v_{h}^{\alpha} \in \mathbb{V}_{h}^{\alpha} \mid v_{h, \nu}^{\alpha}=0 \text { on } \Gamma_{c}\right\}
$$


Let $P_{\alpha, h}: W_{h} \mapsto \mathbb{V}_{h}^{\alpha}, \alpha=1,2$ be the extension operator defined by:

$$
P_{\alpha, h} \varphi_{h}=v_{h}^{\alpha}, \quad \varphi_{h} \in W_{h},
$$

where $v_{h}^{\alpha} \in \mathbb{V}_{h}^{\alpha}$ solves

$$
\begin{array}{ll}
a^{\alpha}\left(v_{h}^{\alpha}, w_{h}^{\alpha}\right)=0 & \forall w_{h}^{\alpha} \in \mathbb{V}_{0, h}^{\alpha}, \\
v_{h, \nu}^{\alpha}=\varphi_{h} & \text { on } \Gamma_{c} .
\end{array}
$$

We define the discrete Poincaré-Steklov operator $S_{\alpha, h}: W_{h} \mapsto\left(W_{h}\right)^{\prime}$ (the dual space of $W_{h}$ ) by

$$
\left\langle S_{\alpha, h} \varphi_{h}, \psi_{h}\right\rangle=a^{\alpha}\left(P_{\alpha, h} \varphi_{h}, v_{h}^{\alpha}\right) \quad \forall v_{h}^{\alpha} \in \mathbb{V}_{h}^{\alpha}, \quad v_{h, \nu}^{\alpha}=(-1)^{\alpha-1} \psi_{h} \text { on } \Gamma_{c} .
$$

Finally, we introduce the mapping $Q_{2, h}: W_{h} \mapsto W_{h}, \lambda_{h} \mapsto Q_{2, h} \lambda_{h}=v_{h, \nu}^{2}$, where $v_{h}^{2} \in \mathbb{K}_{h}^{2}\left(\lambda_{h}-\right.$ $\left.U_{h, \nu}^{2}\right)$ solves

$$
a^{2}\left(v_{h}^{2}, z_{h}^{2}-v_{h}^{2}\right) \geq 0 \quad \forall z_{h}^{2} \in \mathbb{K}_{h}^{2}\left(\lambda_{h}-U_{h, \nu}^{2}\right) .
$$

Here $U_{h}^{2} \in \mathbb{V}_{h}^{2}$ is the finite element solution of (4.7) and for any $\varphi_{h} \in W_{h}$

$$
\mathbb{K}_{h}^{2}\left(\varphi_{h}\right)=\left\{v_{h}^{2} \in \mathbb{V}_{h}^{2} \mid \varphi_{h}-v_{h, \nu}^{2} \leq 0 \text { on } \Gamma_{c}\right\}, \quad \lambda_{h} \in W_{h} .
$$

To prove convergence in the discrete case, we shall suppose that the extension operators $P_{\alpha, h}$ : $W_{h} \mapsto \mathbb{V}_{h}^{\alpha}$ enjoy the following property:

$$
\left\|P_{\alpha, h} \varphi_{h}\right\|_{1, \Omega^{\alpha}} \leq C\left\|\varphi_{h}\right\|_{\frac{1}{2}} \quad \forall \varphi_{h} \in W_{h}
$$

with $C>0$ independent of $h$. From this we obtain the following statement.

Lemma 4. Let $(\mathbb{A})$ be satisfied. Then

$$
\begin{aligned}
\left|\left\langle S_{\alpha, h} q_{h}, \varphi_{h}\right\rangle\right| & \leq C_{1}\left\|q_{h}\right\|_{\frac{1}{2}}\left\|\varphi_{h}\right\|_{\frac{1}{2}}, \\
(-1)^{\alpha-1}\left\langle S_{\alpha, h} q_{h}, q_{h}\right\rangle & \geq C_{2}\left\|q_{h}\right\|_{\frac{1}{2}}^{2}
\end{aligned}
$$

hold for every $q_{h}, \varphi_{h} \in W_{h}$, where $C_{1}>0$ and $C_{2}>0$ are independent of $h, \alpha=1,2$.

Proof. Let $q_{h}, \varphi_{h} \in W_{h}$ be given and $v_{h}^{\alpha}=P_{\alpha, h} \varphi_{h} \in \mathbb{V}_{h}^{\alpha}$. From the definition of $S_{\alpha, h}$ and (A) we have:

$$
\begin{aligned}
\left|\left\langle S_{\alpha, h} q_{h}, \varphi_{h}\right\rangle\right| & =\left|a^{\alpha}\left(P_{\alpha, h} q_{h}, P_{\alpha, h} \varphi_{h}\right)\right| \\
& \leq\left\|a^{\alpha}\right\|\left\|P_{\alpha, h} q_{h}\right\|_{1, \Omega^{\alpha}}\left\|P_{\alpha, h} \varphi_{h}\right\|_{1, \Omega^{\alpha}} \\
& \leq C_{1}\left\|q_{h}\right\|_{\frac{1}{2}}\left\|\varphi_{h}\right\|_{\frac{1}{2}}
\end{aligned}
$$

proving (5.1). Further

$$
\left\langle S_{\alpha, h} \varphi_{h}, \varphi_{h}\right\rangle=a^{\alpha}\left(P_{\alpha, h} \varphi_{h},(-1)^{\alpha-1} P_{\alpha, h} \varphi_{h}\right) \quad \forall \varphi_{h} \in W_{h}
$$

using that $(-1)^{\alpha-1}\left(P_{\alpha, h} \varphi_{h}\right) \cdot \nu=(-1)^{\alpha-1} \varphi_{h}$ on $\Gamma_{c}$. From this we arrive at (5.2). 
Remark 5. Analogous results hold for the operators $S_{\alpha, h}^{-1}, \alpha=1,2$.

Theorem 6. The discrete operator $S_{2, h} Q_{2, h}: W_{h} \mapsto W_{h}$ is Lipschitz and monotone:

$$
\begin{aligned}
& \exists l>0: \quad\left\|S_{2, h} Q_{2, h} \varphi_{h}-S_{2, h} Q_{2, h} \psi_{h}\right\|_{-\frac{1}{2}} \leq l\left\|\varphi_{h}-\psi_{h}\right\|_{\frac{1}{2}}, \\
& \left\langle S_{2, h} Q_{2, h} \varphi_{h}-S_{2, h} Q_{2, h} \psi_{h}, \varphi_{h}-\psi_{h}\right\rangle \leq 0
\end{aligned}
$$

hold for every $\varphi_{h}, \psi_{h} \in W_{h}$. In addition, if $(\mathbb{A})$ is satisfied then l in (5.4) does not depend on $h$.

Proof. Let $\varphi_{h}, \psi_{h} \in W_{h}$ be given and $u_{h}^{2} \in \mathbb{K}_{h}^{2}\left(\varphi_{h}-U_{h, \nu}^{2}\right), v_{h}^{2} \in \mathbb{K}_{h}^{2}\left(\psi_{h}-U_{h, \nu}^{2}\right)$ be the unique solutions of

$$
a^{2}\left(u_{h}^{2}, z_{h}^{2}-u_{h}^{2}\right) \geq 0 \quad \forall z_{h}^{2} \in \mathbb{K}_{h}^{2}\left(\varphi_{h}-U_{h, \nu}^{2}\right)
$$

and

$$
a^{2}\left(v_{h}^{2}, z_{h}^{2}-v_{h}^{2}\right) \geq 0 \quad \forall z_{h}^{2} \in \mathbb{K}_{h}^{2}\left(\psi_{h}-U_{h, \nu}^{2}\right) .
$$

It is easily seen that the convex sets $\mathbb{K}_{h}^{2}\left(\varphi_{h}-U_{h, \nu}^{2}\right)$ and $\mathbb{K}_{h}^{2}\left(\psi_{h}-U_{h, \nu}^{2}\right)$ can be written as

$$
\begin{aligned}
& \mathbb{K}_{h}^{2}\left(\varphi_{h}-U_{h, \nu}^{2}\right)=\Phi_{h}-U_{h}^{2}+\mathbb{K}_{h}^{2}(0), \\
& \mathbb{K}_{h}^{2}\left(\psi_{h}-U_{h, \nu}^{2}\right)=\Psi_{h}-U_{h}^{2}+\mathbb{K}_{h}^{2}(0),
\end{aligned}
$$

respectively, where $\Phi_{h}=P_{2, h} \varphi_{h}, \Psi_{h}=P_{2, h} \psi_{h}$. In other words: any $z_{h}^{2} \in \mathbb{K}_{h}^{2}\left(\varphi_{h}-U_{h, \nu}^{2}\right)$ can be written in the form

$$
z_{h}^{2}=\Phi_{h}-U_{h}^{2}+\bar{z}_{h}^{2}
$$

for an appropriate $\bar{z}_{h}^{2} \in \mathbb{K}_{h}^{2}(0)$ (analogously for elements of $\mathbb{K}_{h}^{2}\left(\psi_{h}-U_{h, \nu}^{2}\right)$ ). Hence the solutions $u_{h}^{2}, v_{h}^{2}$ of (5.6) and (5.7), respectively, can be expressed as

$$
\left.\begin{array}{l}
u_{h}^{2}=\Phi_{h}-U_{h}^{2}+\bar{u}_{h}^{2}, \\
v_{h}^{2}=\Psi_{h}-U_{h}^{2}+\bar{v}_{h}^{2},
\end{array}\right\}
$$

where $\bar{u}_{h}^{2}, \bar{v}_{h}^{2} \in \mathbb{K}_{h}^{2}(0)$ are the solutions of

$$
\begin{aligned}
& a^{2}\left(\bar{u}_{h}^{2}, \bar{z}_{h}^{2}-\bar{u}_{h}^{2}\right) \geq a^{2}\left(U_{h}^{2}-\Phi_{h}, \bar{z}_{h}^{2}-\bar{u}_{h}^{2}\right), \\
& a^{2}\left(\bar{v}_{h}^{2}, \bar{z}_{h}^{2}-\bar{v}_{h}^{2}\right) \geq a^{2}\left(U_{h}^{2}-\Psi_{h}, \bar{z}_{h}^{2}-\bar{v}_{h}^{2}\right)
\end{aligned}
$$

for every $\bar{z}_{h}^{2} \in \mathbb{K}_{h}^{2}(0)$. Inserting $\bar{z}_{h}^{2}:=\bar{v}_{h}^{2}$ into (5.10) and $\bar{z}_{h}^{2}:=\bar{u}_{h}^{2}$ into (5.11) and summing both inequalities, we obtain

$$
\begin{aligned}
\gamma\left\|\bar{u}_{h}^{2}-\bar{v}_{h}^{2}\right\|_{1, \Omega^{2}}^{2} & \leq a^{2}\left(\bar{u}_{h}^{2}-\bar{v}_{h}^{2}, \bar{u}_{h}^{2}-\bar{v}_{h}^{2}\right) \leq a^{2}\left(\Psi_{h}-\Phi_{h}, \bar{u}_{h}^{2}-\bar{v}_{h}^{2}\right) \\
& \leq c\left\|\Psi_{h}-\Phi_{h}\right\|_{1, \Omega^{2}}\left\|\bar{u}_{h}^{2}-\bar{v}_{h}^{2}\right\|_{1, \Omega^{2}}
\end{aligned}
$$


From this and (5.9) we have:

$$
\left\|u_{h}^{2}-v_{h}^{2}\right\|_{1, \Omega^{2}} \leq c\left\|\Psi_{h}-\Phi_{h}\right\|_{1, \Omega^{2}}
$$

where $c:=$ const. $>0$ does not depend on $h$. The definition of $Q_{2, h}$ and the trace theorem yield:

$$
\begin{aligned}
\left\|Q_{2, h} \varphi_{h}-Q_{2, h} \psi_{h}\right\|_{\frac{1}{2}} & =\left\|u_{h, \nu}^{2}-v_{h, \nu}^{2}\right\|_{\frac{1}{2}} \leq c\left\|u_{h}^{2}-v_{h}^{2}\right\|_{1, \Omega^{2}} \\
& \leq c\left\|\Psi_{h}-\Phi_{h}\right\|_{1, \Omega^{2}}=c\left\|P_{2, h}\left(\psi_{h}-\varphi_{h}\right)\right\|_{1, \Omega^{2}}
\end{aligned}
$$

with $c>0$ independent of $h$, again. Finally,

$$
\begin{aligned}
\left\|S_{2, h} Q_{2, h} \varphi_{h}-S_{2, h} Q_{2, h} \psi_{h}\right\|_{-\frac{1}{2}} & \leq\left\|S_{2, h}\right\|\left\|Q_{2, h} \varphi_{h}-Q_{2, h} \psi_{h}\right\|_{\frac{1}{2}} \leq c\left\|S_{1, h}\right\|\left\|P_{2, h}\left(\varphi_{h}-\psi_{h}\right)\right\|_{1, \Omega^{2}} \\
& \leq l\left\|\varphi_{h}-\psi_{h}\right\|_{\frac{1}{2}}
\end{aligned}
$$

for some $l>0$. If $(\mathbb{A})$ is satisfied then $l$ does not depend on $h$ as follows from Lemma 4.

To prove (5.5) let us observe first that from (5.10) it follows:

$$
a^{2}\left(u_{h}^{2}, \Phi_{h}-U_{h}^{2}-u_{h}^{2}\right)=0, \quad a^{2}\left(u_{h}^{2}, \bar{z}_{h}^{2}\right) \geq 0 \quad \forall \bar{z}_{h}^{2} \in \mathbb{K}_{h}^{2}(0)
$$

(analogously for $v_{h}^{2}$ ). Let $\varphi_{h}, \psi_{h} \in W_{h}$ be arbitrary. Then

$$
\begin{aligned}
\left\langle S_{2, h} Q_{2, h} \varphi_{h}-S_{2, h} Q_{2, h} \psi_{h}, \varphi_{h}-\psi_{h}\right\rangle & =a^{2}\left(P_{2, h} Q_{2, h} \varphi_{h}-P_{2, h} Q_{2, h} \psi_{h}, P_{2, h} \psi_{h}-P_{2, h} \varphi_{h}\right) \\
& =a^{2}\left(u_{h}^{2}-v_{h}^{2}, \Psi_{h}-\Phi_{h}\right),
\end{aligned}
$$

where $u_{h}^{2}, v_{h}^{2}$ are the solutions of (5.6) and (5.7), respectively. The last term in (5.13) is nonpositive. Indeed,

$$
\begin{aligned}
a^{2}\left(u_{h}^{2}-v_{h}^{2}, \Psi_{h}-\Phi_{h}\right)= & a^{2}\left(u_{h}^{2}-v_{h}^{2}, \Psi_{h}-v_{h}^{2}-U_{h}^{2}\right)+a^{2}\left(u_{h}^{2}-v_{h}^{2}, U_{h}^{2}-\Phi_{h}+u_{h}^{2}\right) \\
& +a^{2}\left(u_{h}^{2}-v_{h}^{2}, v_{h}^{2}-u_{h}^{2}\right) \\
\leq & a^{2}\left(u_{h}^{2}, \Psi_{h}-v_{h}^{2}-U_{h}^{2}\right)-a^{2}\left(v_{h}^{2}, U_{h}^{2}-\Phi_{h}+u_{h}^{2}\right) \\
= & -a^{2}\left(u_{h}^{2}, \bar{v}_{h}^{2}\right)-a^{2}\left(v_{h}^{2}, \bar{u}_{h}^{2}\right) \leq 0,
\end{aligned}
$$

where $\bar{u}_{h}^{2}, \bar{v}_{h}^{2} \in \mathbb{K}_{h}^{2}(0)$ are defined by (5.9). Here we used (5.12) and the ellipticity of the bilinear form $a^{2}$.

Let $T_{\theta, h}: W_{h} \mapsto W_{h}$ be the mapping defined by

$$
T_{\theta, h} \lambda_{h}:=\lambda_{h}-\frac{\theta}{2} M_{h}\left(S_{1, h} \lambda_{h}-S_{2, h} Q_{2, h} \lambda_{h}+g_{0, h}\right),
$$

where $M_{h}:=S_{1, h}^{-1}-S_{2, h}^{-1}, g_{0, h}:=-S_{2, h} U_{h, \nu}^{2}$ and $\theta>0$. This mapping can be viewed to be a discretization of $T_{\theta}$ introduced in Section 4. 
Theorem 7. Let $(\mathbb{A})$ be satisfied. Then there exists $\theta_{0}>0$ which does not depend on $h$ such that $T_{\theta, h}$ is contractive for any $\theta \in\left(0, \theta_{0}\right)$.

Proof. It can be done in the same way as the one of Theorem 3 using (5.4) and (5.5).

Corollary 8. There exists a unique fixed point $\bar{\lambda}_{h}$ of $T_{\theta, h}$ in $W_{h}, \theta \in\left(0, \theta_{0}\right)$, and the method of successive approximations is convergent for any choice of the initial approximation $\lambda_{h, 0} \in W_{h}$.

Let us comment on the satisfaction of $(\mathbb{A})$ (see also [4]). Recall that the extension mapping $P_{\alpha}: H^{1 / 2}\left(\Gamma_{c}\right) \mapsto W^{\alpha}, \alpha=1,2$ is defined by (3.2) and $P_{\alpha, h}: W_{h} \mapsto \mathbb{V}_{h}^{\alpha}$ by the finite element approximation of (3.2): $\varphi_{h} \in W_{h}$ given, the extension $u_{h}^{\alpha}:=P_{\alpha, h} \varphi_{h} \in \mathbb{V}_{h}^{\alpha}$ solves

$$
\left.\begin{array}{cl}
a^{\alpha}\left(u_{h}^{\alpha}, w_{h}^{\alpha}\right)=0 & \forall w_{h}^{\alpha} \in \mathbb{V}_{0, h}^{\alpha}, \\
u_{h, \nu}^{\alpha}=\varphi_{h} & \text { on } \Gamma_{c} .
\end{array}\right\}
$$

Next we shall suppose that the following assumptions are satisfied:

(i) (regularity assumption)

there exists $\varepsilon \in(0,1 / 2)$ such that for any $\varphi \in H^{1 / 2+\varepsilon}\left(\Gamma_{c}\right)$ the solution $u^{\alpha}$ of (3.2) belongs to $\left(H^{1+\varepsilon}\left(\Omega^{\alpha}\right)\right)^{2}$ and

$$
\exists c:=c(\varepsilon)>0:\left\|u^{\alpha}\right\|_{1+\varepsilon, \Omega^{\alpha}} \leq c\|\varphi\|_{1 / 2+\varepsilon, \Gamma_{c}} \quad \forall \varphi \in H^{1 / 2+\varepsilon}\left(\Gamma_{c}\right) ;
$$

(ii) (inverse inequality in $W_{h}$ )

$$
\exists c>0:\left\|\varphi_{h}\right\|_{1 / 2+\varepsilon} \leq c h^{-\varepsilon}\left\|\varphi_{h}\right\|_{1 / 2} \quad \forall \varphi_{h} \in W_{h} \quad \forall h>0 .
$$

Proposition 9. Under (i) and (ii), the assumption $(\mathbb{A})$ is satisfied.

Proof. Let $\varphi_{h} \in W_{h}$ be given and $u_{h}^{\alpha}=P_{\alpha, h} \varphi_{h}$. Then

$$
\left\|u_{h}^{\alpha}\right\|_{1, \Omega^{\alpha}} \leq\left\|u^{\alpha}\right\|_{1, \Omega^{\alpha}}+\left\|u_{h}^{\alpha}-u^{\alpha}\right\|_{1, \Omega^{\alpha}} \leq c\left\{\left\|\varphi_{h}\right\|_{1 / 2}+\inf _{w_{h}^{\alpha} \in \widetilde{\mathbb{V}}_{h}^{\alpha}}\left\|u^{\alpha}-w_{h}^{\alpha}\right\|_{1, \Omega^{\alpha}}\right\},
$$

where $c>0$ does not depend on $h>0$ and

$$
\widetilde{\mathbb{V}}_{h}^{\alpha}=\left\{w_{h}^{\alpha} \in V_{h}^{\alpha} \mid w_{h, \nu}^{\alpha}=\varphi_{h} \text { on } \Gamma_{c}\right\} .
$$

Denote $r_{h}^{\alpha}:\left(H^{1+\varepsilon}\left(\Omega^{\alpha}\right)\right)^{2} \cap \mathbb{V}^{\alpha}: \mapsto \mathbb{V}_{h}^{\alpha}$ the piecewise linear Lagrange interpolation operator. Since $r_{h}^{\alpha} u^{\alpha} \in \widetilde{\mathbb{V}}_{h}^{\alpha}$, we can use $w_{h}^{\alpha}:=r_{h}^{\alpha} u^{\alpha}$ on the right of (5.15). Then

$$
\left\|u^{\alpha}-r_{h} u_{h}^{\alpha}\right\|_{1, \Omega^{\alpha}} \leq c h^{\varepsilon}\|u\|_{1+\varepsilon, \Omega^{\alpha}} \leq c h^{\varepsilon}\left\|\varphi_{h}\right\|_{1 / 2+\varepsilon} .
$$




\section{Implementation}

In this section we describe in more details the finite element approximation of the problem and its implementation. We start with the algebraic formulation of the original non-decomposed problem.

Let $n_{\alpha}:=\operatorname{dim} \mathbb{V}_{0, h}^{\alpha}, \alpha=1,2$ and $n:=n_{1}+n_{2}$. Further, let $m$ be the number of the contact nodes of $\Omega^{1}$, i.e., the nodes of $\mathcal{T}_{h}^{1}$ lying on $\bar{\Gamma}_{c}^{1} \backslash \bar{\Gamma}_{u}^{1}$. As we consider the matching grids, the contact nodes of $\Omega^{1}$ and $\Omega^{2}$ coincide when $\Gamma_{c}^{1}=\Gamma_{c}^{2}$. If $\Gamma_{c}^{1} \neq \Gamma_{c}^{2}$, the contact nodes of $\Omega^{1}$ are bijectively mapped onto the ones of $\Omega^{2}$ by the transfer mapping $\chi$ as mentioned in Section 2 .

By $\mathbf{A} \in \mathbb{R}^{n \times n}$ and $\mathbf{b} \in \mathbb{R}^{n}$ we denote the stiffness matrix and the load vector, respectively, of the whole structure. Let us note that $\mathbf{A}, \mathbf{b}$ can be naturally decomposed into blocks corresponding to $\Omega^{1}$ and $\Omega^{2}$ so that $\mathbf{A}=\operatorname{diag}\left(\mathbf{A}_{1}, \mathbf{A}_{2}\right), \mathbf{b}=\left(\mathbf{b}_{1}^{\top}, \mathbf{b}_{2}^{\top}\right)^{\top}$, where $\mathbf{A}_{\alpha} \in \mathbb{R}^{n_{\alpha} \times n_{\alpha}}$ are symmetric, positive definite and $\mathbf{b}_{\alpha} \in \mathbb{R}^{n_{\alpha}}, \alpha=1,2$. We introduce the (Dirichlet) trace matrices $\mathbf{B}_{\alpha} \in \mathbb{R}^{2 m \times n_{\alpha}}$, $\alpha=1,2$ having only one non-zero component in each row, namely "1" on the position of a contact displacement component. Moreover, we denote by $\mathbf{N} \in \mathbb{R}^{m \times 2 m}$ the matrix projecting contact displacements to the direction of $\nu$, i.e., each row of $\mathbf{N}$ has at most two non-zero entries given by the coordinates of $\nu$ in the positions of contact displacement components. Let us note that $\mathbf{B}_{1}, \mathbf{B}_{2}$ and $\mathbf{N}$ have full row-ranks. Finally, suppose that $\mathbf{g} \in \mathbb{R}^{m}$ collects the values of the initial gap at the contact nodes.

The finite element approximation of $(\mathcal{P})$ leads to the following quadratic programming problem:

$$
\begin{aligned}
& \text { minimize } \frac{1}{2} \mathbf{u}^{\top} \mathbf{A u}-\mathbf{u}^{\top} \mathbf{b} \\
& \text { subject to } \mathbf{N B}_{1} \mathbf{u}_{1}-\mathbf{N B}_{2} \mathbf{u}_{2} \leq \mathbf{g},
\end{aligned}
$$

where $\mathbf{u}=\left(\mathbf{u}_{1}^{\top}, \mathbf{u}_{2}^{\top}\right)^{\top}, \mathbf{u}_{\alpha} \in \mathbb{R}^{n_{\alpha}}, \alpha=1,2$.

The problem (6.1) can be solved by the following discrete counterpart of ALGORITHM 3.1.

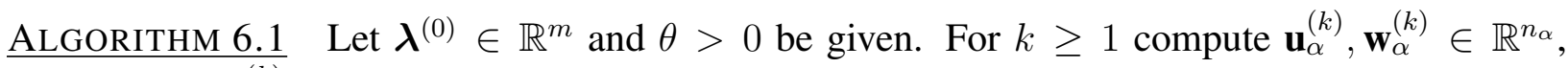
$\overline{\alpha=1,2 \text { and } \boldsymbol{\lambda}^{(k)}} \in \mathbb{R}^{m}$ as follows:

(Step 1) $\mathbf{u}_{1}^{(k)}:=\operatorname{argmin} \frac{1}{2} \mathbf{u}_{1}^{\top} \mathbf{A}_{1} \mathbf{u}_{1}-\mathbf{u}_{1}^{\top} \mathbf{b}_{1}$ subject to $\mathbf{N B}_{1} \mathbf{u}_{1}=\boldsymbol{\lambda}^{(k-1)}$;

(Step 2) $\mathbf{u}_{2}^{(k)}:=\operatorname{argmin} \frac{1}{2} \mathbf{u}_{2}^{\top} \mathbf{A}_{2} \mathbf{u}_{2}-\mathbf{u}_{2}^{\top} \mathbf{b}_{2}$ subject to $\boldsymbol{\lambda}^{(k-1)}-\mathbf{N B}_{2} \mathbf{u}_{2} \leq \mathbf{g}$;

(Step 3) Solve $\mathbf{A}_{1} \mathbf{w}_{1}^{(k)}=\frac{1}{2} \mathbf{B}_{1}^{\top}\left(\mathbf{B}_{1}\left(\mathbf{b}_{1}-\mathbf{A}_{1} \mathbf{u}_{1}^{(k)}\right)-\mathbf{B}_{2}\left(\mathbf{A}_{2} \mathbf{u}_{2}^{(k)}-\mathbf{b}_{2}\right)\right)$;

(Step 4) Solve $\mathbf{A}_{2} \mathbf{w}_{2}^{(k)}=-\frac{1}{2} \mathbf{B}_{2}^{\top}\left(\mathbf{B}_{1}\left(\mathbf{b}_{1}-\mathbf{A}_{1} \mathbf{u}_{1}^{(k)}\right)-\mathbf{B}_{2}\left(\mathbf{A}_{2} \mathbf{u}_{2}^{(k)}-\mathbf{b}_{2}\right)\right)$;

(Step 5) $\boldsymbol{\lambda}^{(k)}=\boldsymbol{\lambda}^{(k-1)}+\theta\left(\mathbf{N B}_{1} \mathbf{w}_{1}^{(k)}-\mathbf{N B}_{2} \mathbf{w}_{2}^{(k)}\right)$.

We shall show that the dual formulation simplifies considerably the implementation of the algorithm. The minimization problem in Step 1 is equivalent to solving the following saddle-point system:

$$
\left(\begin{array}{cc}
\mathbf{A}_{1} & \mathbf{B}_{1}^{\top} \mathbf{N}^{\top} \\
\mathbf{N B}_{1} & \mathbf{O}
\end{array}\right)\left(\begin{array}{c}
\mathbf{u}_{1} \\
\mathbf{s}_{1}
\end{array}\right)=\left(\begin{array}{c}
\mathbf{b}_{1} \\
\boldsymbol{\lambda}^{(k-1)}
\end{array}\right)
$$


Eliminating the first unknown $\mathbf{u}_{1}$, we obtain:

$$
\mathbf{C}_{1} \mathbf{s}_{1}=\mathbf{d}_{1}
$$

where $\mathbf{C}_{1}:=\mathbf{N B}_{1} A_{1}^{-1} B_{1}^{\top} \mathbf{N}^{\top}$ and $\mathbf{d}_{1}:=\mathbf{N B}_{1} A_{1}^{-1} \mathbf{b}_{1}-\boldsymbol{\lambda}^{(k-1)}$. Since $\mathbf{C}_{1}$ is symmetric, positive definite, the system (6.2) can be solved by the conjugate gradient method [9].

The constrained minimization in Step 2 is equivalent to the saddle-point problem:

$$
\text { Find }\left(\mathbf{u}_{2}, \mathbf{s}_{2}\right) \in \mathbb{R}^{n_{2}} \times \mathbb{R}_{+}^{m}: \quad \mathcal{L}\left(\mathbf{u}_{2}, \mathbf{s}_{2}\right)=\min _{\mathbf{v}_{2} \in \mathbb{R}^{n_{2}}} \max _{\mathbf{r}_{2} \in \mathbb{R}_{+}^{m}} \mathcal{L}\left(\mathbf{v}_{2}, \mathbf{r}_{2}\right)=\max _{\mathbf{r}_{2} \in \mathbb{R}_{+}^{m}} \min _{\mathbf{v}_{2} \in \mathbb{R}^{n_{2}}} \mathcal{L}\left(\mathbf{v}_{2}, \mathbf{r}_{2}\right),
$$

where $\mathcal{L}: \mathbb{R}^{n_{2}} \times \mathbb{R}_{+}^{m} \mapsto \mathbb{R}$ is the Lagrangian defined by

$$
\mathcal{L}\left(\mathbf{v}_{2}, \mathbf{r}_{2}\right):=\frac{1}{2} \mathbf{v}_{2}^{\top} \mathbf{A}_{2} \mathbf{v}_{2}-\mathbf{v}_{2}^{\top} \mathbf{b}_{2}+\mathbf{r}_{2}^{\top}\left(\boldsymbol{\lambda}^{(k-1)}-\mathbf{N B}_{2} \mathbf{v}_{2}-\mathbf{g}\right)
$$

and $\mathbb{R}_{+}^{m}:=\left\{\mathbf{r}_{2} \in \mathbb{R}^{m}: \mathbf{r}_{2} \geq \mathbf{0}\right\}$. The max-min problem leads to the quadratic programming problem with the simple constraints:

$$
\text { minimize } \frac{1}{2} \mathbf{s}_{2}^{\top} \mathbf{C}_{2} \mathbf{s}_{2}-\mathbf{s}_{2}^{\top} \mathbf{d}_{2} \quad \text { subject to } \mathbf{s}_{2} \geq \mathbf{0}
$$

where $\mathbf{C}_{2}:=\mathbf{N B}_{2} \mathbf{A}_{2}^{-1} \mathbf{B}_{2}^{\top} \mathbf{N}^{\top}$ is again symmetric, positive definite and $\mathbf{d}_{2}:=\boldsymbol{\lambda}^{(k-1)}-\mathbf{N B}_{2} \mathbf{A}_{2}^{-1} \mathbf{b}_{2}-$ g. To solve (6.3) one can use the conjugate gradient method combined with the projected gradient technique [6].

Let $\mathbf{s}_{1}^{(k)}, \mathbf{s}_{2}^{(k)}$ be the solutions to (6.2), (6.3), respectively. Then the linear systems in Step 3 and Step 4 read as follows:

$$
\mathbf{A}_{1} \mathbf{w}_{1}^{(k)}=\frac{1}{2} \mathbf{B}_{1}^{\top} \mathbf{N}^{\top}\left(\mathbf{s}_{1}^{(k)}-\mathbf{s}_{2}^{(k)}\right) \quad \text { and } \quad \mathbf{A}_{2} \mathbf{w}_{2}^{(k)}=-\frac{1}{2} \mathbf{B}_{2}^{\top} \mathbf{N}^{\top}\left(\mathbf{s}_{1}^{(k)}-\mathbf{s}_{2}^{(k)}\right) .
$$

Let us note that the actions of $\mathbf{A}_{1}^{-1}$ and $\mathbf{A}_{2}^{-1}$ are evaluated by the backward substitutions based on the Cholesky factorization of $\mathbf{A}_{1}$ and $\mathbf{A}_{2}$, respectively.

Let us denote the relative precision of the $k$-th iterative step of ALGORITHM 6.1 by

$$
\varepsilon_{\lambda}^{(k)}:=\left\|\boldsymbol{\lambda}^{(k)}-\boldsymbol{\lambda}^{(k-1)}\right\|_{\mathbb{R}^{m}} /\left\|\boldsymbol{\lambda}^{(k)}\right\|_{\mathbb{R}^{m}},
$$

where $\|\cdot\|_{\mathbb{R}^{m}}$ is the Euclidean norm in $\mathbb{R}^{m}$. We terminate if

$$
\varepsilon_{\lambda}^{(k)} \leq \text { tol }
$$

for a prescribed tolerance $t o l>0$. In order to increase the efficiency of the algorithm, we initialize the inner iterative solvers in Step 1 and Step 2 by the respective results from the previous outer iterate, i.e. by $\mathbf{s}_{1}^{(k-1)}$ and $\mathbf{s}_{2}^{(k-1)}$, and we terminate them by an adaptive (inner) terminating tolerance tol $l_{i n}^{(k)}>0$. The idea is to choose $\operatorname{tol}_{i n}^{(k)}$ in such a way that it respects the precision $\varepsilon_{\lambda}^{(k-1)}$ achieved in the outer loop:

$$
\operatorname{tol}_{\text {in }}^{(k)}:=r_{\text {tol }} \times \varepsilon_{\lambda}^{(k-1)}
$$


where $0<r_{\text {tol }}<1, \varepsilon_{\lambda}^{(0)}:=1$.

As we shall compare ALGORITHM 6.1 with the original one proposed in [3], we recall its implementation.

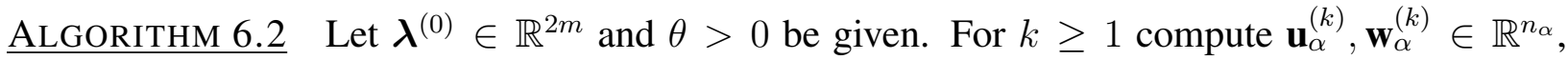
$\alpha=1,2$ and $\boldsymbol{\lambda}^{(k)} \in \mathbb{R}^{2 m}$ as follows:

(Step 1) $\mathbf{u}_{1}^{(k)}:=\operatorname{argmin} \frac{1}{2} \mathbf{u}_{1}^{\top} \mathbf{A}_{1} \mathbf{u}_{1}-\mathbf{u}_{1}^{\top} \mathbf{b}_{1}$ subject to $\mathbf{B}_{1} \mathbf{u}_{1}=\boldsymbol{\lambda}^{(k-1)}$;

(Step 2) $\mathbf{u}_{2}^{(k)}:=\operatorname{argmin} \frac{1}{2} \mathbf{u}_{2}^{\top} \mathbf{A}_{2} \mathbf{u}_{2}-\mathbf{u}_{2}^{\top} \mathbf{b}_{2}$ subject to $\mathbf{N} \boldsymbol{\lambda}^{(k-1)}-\mathbf{N B}_{2} \mathbf{u}_{2} \leq \mathbf{g}$;

(Step 3) Solve $\mathbf{A}_{1} \mathbf{w}_{1}^{(k)}=\frac{1}{2} \mathbf{B}_{1}^{\top}\left(\mathbf{B}_{1}\left(\mathbf{b}_{1}-\mathbf{A}_{1} \mathbf{u}_{1}^{(k)}\right)-\mathbf{B}_{2}\left(\mathbf{A}_{2} \mathbf{u}_{2}^{(k)}-\mathbf{b}_{2}\right)\right)$;

(Step 4) Solve $\mathbf{A}_{2} \mathbf{w}_{2}^{(k)}=-\frac{1}{2} \mathbf{B}_{2}^{\top}\left(\mathbf{B}_{1}\left(\mathbf{b}_{1}-\mathbf{A}_{1} \mathbf{u}_{1}^{(k)}\right)-\mathbf{B}_{2}\left(\mathbf{A}_{2} \mathbf{u}_{2}^{(k)}-\mathbf{b}_{2}\right)\right)$;

(Step 5) $\boldsymbol{\lambda}^{(k)}=\boldsymbol{\lambda}^{(k-1)}+\theta\left(\mathbf{B}_{1} \mathbf{w}_{1}^{(k)}-\mathbf{B}_{2} \mathbf{w}_{2}^{(k)}\right)$.

At the first glance Algorithm 6.1 and Algorithm 6.2 look similar, however, the second one uses vectors from $\mathbb{R}^{2 m}$. Consequently, the solution of Step 1 of ALGORITHM 6.2 based on the dual formulation requires to solve two times larger linear system.

\section{Numerical Experiments}

In this section, we shall compare performances of both ALGORITHM 6.1 and ALGORITHM 6.2 for various values of $\theta$ and degrees of freedom $n$ and $m$. In tables below we report the computational time (time), the number of the outer iterations (out:=final $k$ ), the number of the inner iteration (inn that is the total number of the matrix-vector multiplications by $\mathbf{C}_{1}$ and $\mathbf{C}_{2}$ ), the final precision of the contact displacements $\varepsilon_{\lambda}:=\varepsilon_{\lambda}^{(\text {final } k)}$, the relative continuity of the normal contact stresses ( $n \_$stress) and the relative continuity of the tangential contact stresses ( $t$ sstress). The total efficiency of the algorithms is assessed by the ratio eff $:=i n n / m$. We set $t o l=10^{-5}, r_{\text {tol }}=0.5$ and stop computations if the number of the outer iterations is greater than $\max _{-} i t=100$. All computations are performed in Matlab 7.1 on Intel(R)Core(TM)2 Duo CPU, $2 \mathrm{GHz}$ with 1 GB RAM.

The quantity inn supplies an information on the computational time as it is proportional to inn. The ratio eff represents a comparison of our algorithms with the realization of "similar linear problems" by the standard conjugate gradient method. It is well-known that the number of conjugate gradient iterations (i.e., the number of matrix-vector multiplications) is bounded by the size of the problem. One can say that our algorithms exhibit the complexity comparable with the conjugate gradient method when eff is less than two.

Let us note that the geometry of our model problems is similar to that one depicted in Figure 1 ( $\Gamma_{c}^{1}$ coincides with $\Gamma_{c}^{2}$ in Example 1). All experiments use linear finite element approximation of $(\mathcal{P})$. The triangulations of rectangular domains are defined by cutting rectangles obtained by an equidistant rectangulation. If $\Gamma_{c}^{1}$ is a segment of a circle we define the triangulation of $\Omega^{1}$ by adapting the one introduced for an appropriate rectangular domain (see Figure 3). 
Example 1. Let us consider the plane elastic bodies

$$
\Omega^{1}=(0,3) \times(1,2) \text { and } \Omega^{2}=(0,3) \times(0,1)
$$

made of an isotropic, homogeneous material characterized by the Young modulus $2.1 \times 10^{11}$ and the Poisson ratio 0.277 (steal). The decompositions of $\Gamma^{1}$ and $\Gamma^{2}$ are as follows:

$$
\begin{array}{lll}
\Gamma_{u}^{1}=\{0\} \times(1,2), & \Gamma_{c}^{1}=(0,3) \times\{1\}, & \Gamma_{p}^{1}=\Gamma^{1} \backslash \overline{\Gamma_{u}^{1} \cup \Gamma_{c}^{1}} \\
\Gamma_{u}^{2}=\{0\} \times(0,1), & \Gamma_{c}^{2}=(0,3) \times\{1\}, & \Gamma_{p}^{2}=\Gamma^{2} \backslash \overline{\Gamma_{u}^{2} \cup \Gamma_{c}^{2}}
\end{array}
$$

The volume forces vanish for both bodies. The non-vanishing surface tractions $p^{1}=\left(p_{1}^{1}, p_{2}^{1}\right)$ act on $\Gamma_{p}^{1}$ so that

$$
\begin{aligned}
& p_{1}^{1}(s, 2)=0, p_{2}^{1}(s, 2)=p_{2, L}^{1}+p_{2, R}^{1} s, \quad s \in(0,3), \\
& p_{1}^{1}(3, s)=0, p_{2}^{1}(3, s)=p_{2, B}^{1}(2-s)+p_{2, U}^{1}(s-1), \quad s \in(1,2),
\end{aligned}
$$

where $p_{2, L}^{1}=-7 \times 10^{7}, p_{2, R}^{1}=-1 / 3 \times 10^{7}, p_{2, B}^{1}=4 \times 10^{6}$ and $p_{2, U}^{1}=1.8 \times 10^{7}$.

(a) Triangulation of bodies and applied tractions

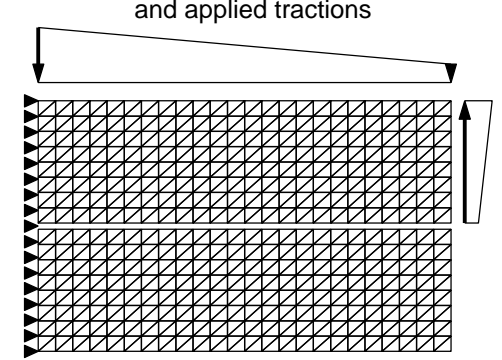

(b) Deformation

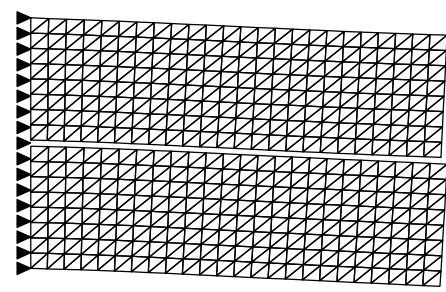

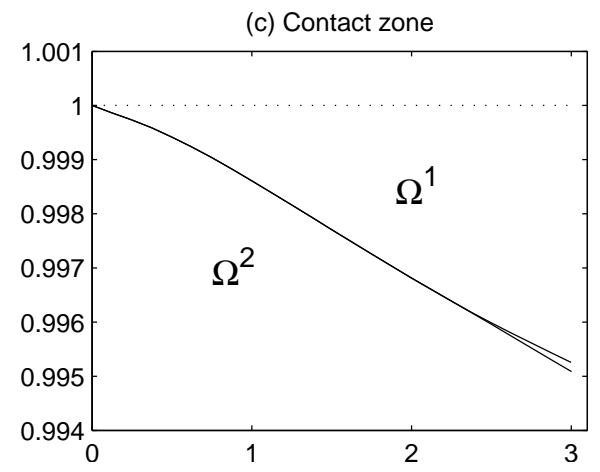

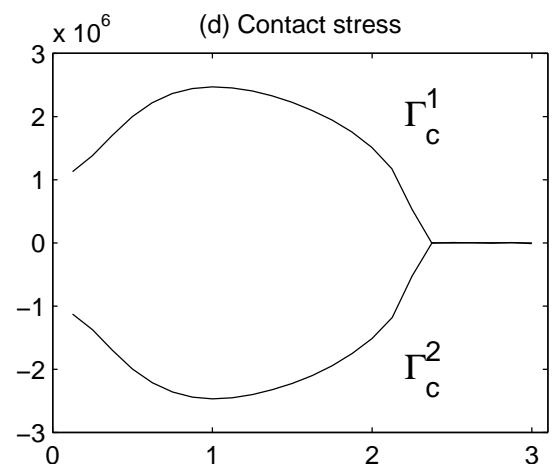

Figure 2: Results for $n=864, m=48$.

Figure 2 shows results of computations. Comparisons of both algorithms for the best choices of $\theta$ are reported in Tables 1 and 2. It is seen that the performance of ALGORITHM 6.1 is considerably 
higher in all cases and, moreover, the continuity of the tangential stresses is comparable to the computer precision. As inn practically does not depend on $m$, the algorithms exhibit the scalability property. In Tables 3 and 4 we demonstrate robustness with respect to $\theta$. The situation is better again for ALGORITHM 6.1 (the symbol "-" means that the terminating tolerance is not achieved in max_it iterations).

Table 1: Algorithm 6.1 for $\theta=0.4$.

\begin{tabular}{rr|rcccc|c}
$n$ & $m$ & time & out/inn & $\varepsilon_{\lambda}$ & $n_{\text {_stress }}$ & t_stress & eff \\
\hline 24 & 6 & 0.01 & $15 / 123$ & $6.4 \mathrm{e}-6$ & $3.7 \mathrm{e}-5$ & $1.6 \mathrm{e}-16$ & 20.50 \\
72 & 12 & 0.02 & $15 / 135$ & $6.6 \mathrm{e}-6$ & $7.2 \mathrm{e}-5$ & $3.9 \mathrm{e}-16$ & 11.25 \\
240 & 24 & 0.03 & $15 / 148$ & $4.1 \mathrm{e}-6$ & $4.8 \mathrm{e}-5$ & $4.9 \mathrm{e}-16$ & 6.17 \\
864 & 48 & 0.11 & $17 / 167$ & $8.0 \mathrm{e}-6$ & $4.5 \mathrm{e}-5$ & $5.0 \mathrm{e}-16$ & 3.48 \\
3264 & 96 & 0.67 & $16 / 168$ & $7.9 \mathrm{e}-6$ & $5.8 \mathrm{e}-5$ & $6.4 \mathrm{e}-16$ & 1.75 \\
12672 & 192 & 6.41 & $18 / 193$ & $2.5 \mathrm{e}-6$ & $1.8 \mathrm{e}-5$ & $6.8 \mathrm{e}-16$ & 1.01 \\
49920 & 384 & 44.41 & $16 / 181$ & $7.7 \mathrm{e}-6$ & $2.4 \mathrm{e}-6$ & $6.8 \mathrm{e}-16$ & 0.47
\end{tabular}

Table 2: Algorithm 6.2 for $\theta=0.2$.

\begin{tabular}{rr|rcccc|c}
$n$ & $m$ & time & out/inn & $\varepsilon_{\lambda}$ & $n_{-}$stress & $t_{-}$stress & eff \\
\hline 24 & 6 & 0.02 & $41 / 301$ & $9.7 \mathrm{e}-6$ & $3.8 \mathrm{e}-5$ & $2.5 \mathrm{e}-5$ & 50.17 \\
72 & 12 & 0.04 & $44 / 338$ & $6.6 \mathrm{e}-6$ & $4.9 \mathrm{e}-5$ & $3.4 \mathrm{e}-5$ & 28.17 \\
240 & 24 & 0.09 & $44 / 350$ & $8.3 \mathrm{e}-6$ & $6.8 \mathrm{e}-5$ & $3.7 \mathrm{e}-5$ & 14.58 \\
864 & 48 & 0.25 & $44 / 364$ & $7.6 \mathrm{e}-6$ & $5.8 \mathrm{e}-5$ & $4.2 \mathrm{e}-5$ & 7.58 \\
3264 & 96 & 1.83 & $48 / 414$ & $9.5 \mathrm{e}-6$ & $5.2 \mathrm{e}-5$ & $3.2 \mathrm{e}-5$ & 4.31 \\
12672 & 192 & 12.32 & $45 / 392$ & $7.7 \mathrm{e}-6$ & $3.4 \mathrm{e}-5$ & $2.5 \mathrm{e}-5$ & 2.04 \\
49920 & 384 & 101.12 & $47 / 432$ & $9.4 \mathrm{e}-6$ & $1.5 \mathrm{e}-5$ & $1.0 \mathrm{e}-5$ & 1.13
\end{tabular}

Example 2. Let us modify the geometry of the body $\Omega^{1}$ from the previous example so that

$$
\begin{aligned}
\Omega^{1} & =\left\{\left(x_{1}, x_{2}\right) \in \mathbb{R}^{2}: 0<x_{1}<3,1+c\left(x_{1}\right)<x_{2}<2\right\} \\
\Gamma_{c}^{1} & =\left\{\left(x_{1}, x_{2}\right) \in \mathbb{R}^{2}: 0<x_{1}<3,1+c\left(x_{1}\right)=x_{2}\right\}
\end{aligned}
$$

where $c$ is the segment of the circle defined by $c\left(x_{1}\right)=10.25-\sqrt{9.25^{2}-x_{1}^{2}}$. The definition of $\Gamma_{u}^{1}, \Gamma_{p}^{1}$ and the geometry of $\Omega^{2}$ are the same as in Example 1. The surface tractions $p^{1}=\left(p_{1}^{1}, p_{2}^{1}\right)$ acting on $\Gamma_{p}^{1}$ are defined by

$$
\begin{aligned}
& p_{1}^{1}(s, 2)=0, p_{2}^{1}(s, 2)=p_{2, L}^{1}+p_{2, R}^{1} s, \quad s \in(0,3), \\
& p_{1}^{1}(3, s)=0, p_{2}^{1}(3, s)=p_{2, B}^{1}(2-s)+p_{2, U}^{1}(s-1-c(3)), \quad s \in(1+c(3), 2),
\end{aligned}
$$


Table 3: Algorithm 6.1, out/inn for various $\theta$.

\begin{tabular}{rr|ccccccc}
$n$ & $m$ & $\theta=0.1$ & $\theta=0.2$ & $\theta=0.3$ & $\theta=0.4$ & $\theta=0.5$ & $\theta=0.6$ & $\theta=0.7$ \\
\hline 24 & 6 & $46 / 307$ & $23 / 166$ & $14 / 114$ & $15 / 123$ & $19 / 156$ & $18 / 145$ & $37 / 290$ \\
72 & 12 & $47 / 336$ & $32 / 241$ & $22 / 178$ & $15 / 135$ & $17 / 155$ & $21 / 187$ & $39 / 326$ \\
240 & 24 & $45 / 323$ & $31 / 249$ & $23 / 199$ & $15 / 148$ & $19 / 179$ & $23 / 205$ & $51 / 461$ \\
864 & 48 & $50 / 381$ & $29 / 247$ & $23 / 208$ & $17 / 167$ & $18 / 176$ & $31 / 279$ & $57 / 507$ \\
3264 & 96 & $49 / 383$ & $29 / 251$ & $24 / 231$ & $16 / 168$ & $18 / 187$ & $24 / 235$ & $51 / 486$ \\
12672 & 192 & $49 / 391$ & $30 / 276$ & $23 / 231$ & $18 / 193$ & $18 / 186$ & $25 / 257$ & $63 / 616$ \\
49920 & 384 & $48 / 388$ & $30 / 283$ & $25 / 257$ & $16 / 181$ & $17 / 181$ & $25 / 257$ & $61 / 768$
\end{tabular}

Table 4: Algorithm 6.2, out/inn for various $\theta$.

\begin{tabular}{rr|ccccc}
$n$ & $m$ & $\theta=0.1$ & $\theta=0.2$ & $\theta=0.3$ & $\theta=0.4$ & $\theta=0.5$ \\
\hline 24 & 6 & $81 / 550$ & $41 / 301$ & $27 / 204$ & $23 / 184$ & $44 / 334$ \\
72 & 12 & $84 / 590$ & $44 / 338$ & $33 / 278$ & - & - \\
240 & 24 & $84 / 602$ & $44 / 350$ & $45 / 385$ & - & - \\
864 & 48 & $82 / 613$ & $44 / 364$ & $58 / 489$ & - & - \\
3264 & 96 & $85 / 649$ & $48 / 414$ & $74 / 605$ & - & - \\
12672 & 192 & $83 / 646$ & $45 / 392$ & $62 / 548$ & - & - \\
49920 & 384 & $82 / 645$ & $47 / 432$ & $64 / 570$ & - & -
\end{tabular}

Table 5: Algorithm 6.1 for $\theta=0.3$.

\begin{tabular}{rr|rcccc|c}
$n$ & $m$ & time & out/inn & $\varepsilon_{\lambda}$ & $n_{\text {_stress }}$ & t_stress & eff \\
\hline 24 & 6 & 0.01 & $31 / 210$ & $9.9 \mathrm{e}-6$ & $4.0 \mathrm{e}-5$ & $1.3 \mathrm{e}-16$ & 35.00 \\
72 & 12 & 0.02 & $31 / 256$ & $7.5 \mathrm{e}-6$ & $2.1 \mathrm{e}-5$ & $6.9 \mathrm{e}-16$ & 21.33 \\
240 & 24 & 0.09 & $41 / 375$ & $9.3 \mathrm{e}-6$ & $7.4 \mathrm{e}-5$ & $6.1 \mathrm{e}-16$ & 15.63 \\
864 & 48 & 0.25 & $38 / 361$ & $9.7 \mathrm{e}-6$ & $5.2 \mathrm{e}-5$ & $1.1 \mathrm{e}-15$ & 7.52 \\
3264 & 96 & 1.45 & $40 / 421$ & $7.6 \mathrm{e}-6$ & $2.4 \mathrm{e}-5$ & $9.3 \mathrm{e}-16$ & 4.39 \\
12672 & 192 & 13.01 & $38 / 433$ & $8.7 \mathrm{e}-6$ & $2.2 \mathrm{e}-5$ & $8.2 \mathrm{e}-16$ & 2.26 \\
49920 & 384 & 142.31 & $51 / 634$ & $8.8 \mathrm{e}-6$ & $9.9 \mathrm{e}-6$ & $1.0 \mathrm{e}-15$ & 1.65
\end{tabular}


where $p_{2, L}^{1}=-7 \times 10^{8}, p_{2, R}^{1}=-1 / 3 \times 10^{7}, p_{2, B}^{1}=-4 /(1-c(3)) \times 10^{6}$ and $p_{2, U}^{1}=-4 /(1-$ $c(3)) \times 10^{7}$. The results of computations are shown in Figure 3. In Tables 5-8 we present analogous comparisons as in Example 1 (with max_it=200). The conclusions are similar but the superiority of ALGORITHM 6.1 is now less expressive. The small number of iterations for $n=24,72$ and some $\theta$ corresponds to the wrong (zero) approximation of the contact stress on the coarsest meshes.

(a) Triangulation of bodies and applied tractions

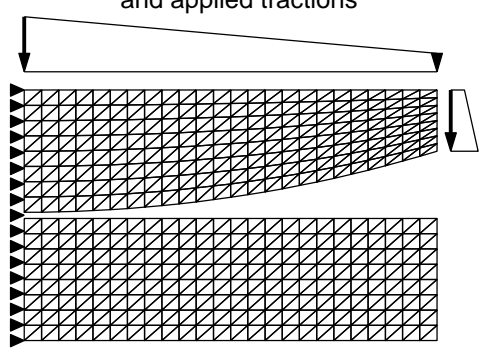

(c) Contact zone

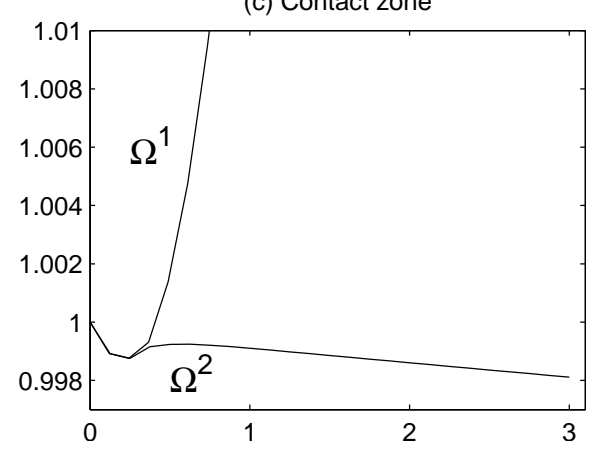

(b) Deformation

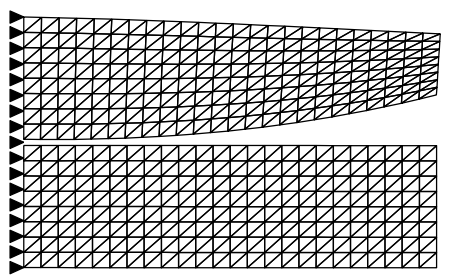

Figure 3: Results for $n=864, m=48$.

\section{Conclusions}

The paper analyzes the new variant of the domain decomposition algorithm for solving frictionless contact problems. Convergence is proved in the continuous as well as discrete setting using the Banach fixed point theorem and, in addition, it is shown its independence on the discretization parameter $h$.

The algorithm can be conceived as a modification of the one proposed in [3]. The main difference consists in the fact that the tangential contact stress is not treated iteratively as it vanishes a-priori. Consequently, the smaller subproblems have to be solved during the iterative process and the algorithm exhibits a better behavior than the original one.

The analysis of the paper can be extended to contact problems with friction. 
Table 6: Algorithm 6.2 for $\theta=0.3$.

\begin{tabular}{rr|rcccc|c}
$n$ & $m$ & time & out/inn & $\varepsilon_{\lambda}$ & $n_{\text {stress }}$ & $t_{\text {stress }}$ & eff \\
\hline 24 & 6 & 0.03 & $45 / 396$ & $9.6 \mathrm{e}-6$ & $2.6 \mathrm{e}-5$ & $2.5 \mathrm{e}-5$ & 66.00 \\
72 & 12 & 0.06 & $50 / 462$ & $9.7 \mathrm{e}-6$ & $1.1 \mathrm{e}-5$ & $1.1 \mathrm{e}-5$ & 38.50 \\
240 & 24 & 0.09 & $50 / 441$ & $8.8 \mathrm{e}-6$ & $5.3 \mathrm{e}-6$ & $4.8 \mathrm{e}-6$ & 18.38 \\
864 & 48 & 0.34 & $51 / 513$ & $5.6 \mathrm{e}-6$ & $1.7 \mathrm{e}-5$ & $1.1 \mathrm{e}-5$ & 10.69 \\
3264 & 96 & 2.54 & $52 / 583$ & $7.3 \mathrm{e}-6$ & $3.8 \mathrm{e}-5$ & $5.7 \mathrm{e}-6$ & 6.07 \\
12672 & 192 & 18.97 & $52 / 635$ & $6.1 \mathrm{e}-6$ & $2.7 \mathrm{e}-5$ & $5.2 \mathrm{e}-6$ & 3.31 \\
49920 & 384 & 151.52 & $50 / 680$ & $9.1 \mathrm{e}-6$ & $5.3 \mathrm{e}-5$ & $1.7 \mathrm{e}-5$ & 1.77
\end{tabular}

Table 7: Algorithm 6.1, out/inn for various $\theta$.

\begin{tabular}{rr|ccccccc}
$n$ & $m$ & $\theta=0.1$ & $\theta=0.2$ & $\theta=0.3$ & $\theta=0.4$ & $\theta=0.5$ & $\theta=0.6$ & $\theta=0.7$ \\
\hline 24 & 6 & $93 / 614$ & $48 / 322$ & $31 / 210$ & $24 / 164$ & $18 / 123$ & $15 / 102$ & $12 / 83$ \\
72 & 12 & $92 / 693$ & $47 / 372$ & $31 / 256$ & $23 / 190$ & $18 / 152$ & $14 / 119$ & $12 / 104$ \\
240 & 24 & $88 / 685$ & $50 / 394$ & $41 / 375$ & $49 / 469$ & $89 / 893$ & - & - \\
864 & 48 & $91 / 751$ & $46 / 404$ & $38 / 361$ & $48 / 492$ & $81 / 877$ & $174 / 1938$ & - \\
3264 & 96 & $87 / 776$ & $47 / 479$ & $40 / 421$ & $48 / 510$ & $73 / 803$ & $140 / 1566$ & $87 / 1040$ \\
12672 & 192 & $87 / 837$ & $46 / 489$ & $38 / 433$ & $66 / 778$ & $79 / 910$ & $122 / 1480$ & $90 / 1070$ \\
49920 & 384 & $87 / 890$ & $44 / 505$ & $51 / 634$ & $92 / 1103$ & $106 / 1270$ & $131 / 1618$ & $122 / 1499$
\end{tabular}

Table 8: AlgORITHM 6.2, out/inn for various $\theta$.

\begin{tabular}{rr|ccccc}
$n$ & $m$ & $\theta=0.1$ & $\theta=0.2$ & $\theta=0.3$ & $\theta=0.4$ & $\theta=0.5$ \\
\hline 24 & 6 & $128 / 1084$ & $67 / 582$ & $45 / 396$ & $34 / 300$ & $27 / 241$ \\
72 & 12 & $140 / 1172$ & $74 / 653$ & $50 / 462$ & $38 / 360$ & $31 / 304$ \\
240 & 24 & $143 / 1136$ & $72 / 571$ & $50 / 441$ & $37 / 423$ & - \\
864 & 48 & $147 / 1350$ & $75 / 701$ & $51 / 513$ & $44 / 492$ & - \\
3264 & 96 & $146 / 1392$ & $75 / 796$ & $52 / 583$ & $63 / 729$ & - \\
12672 & 192 & $145 / 1504$ & $77 / 899$ & $52 / 635$ & $66 / 835$ & - \\
49920 & 384 & $143 / 1579$ & $72 / 929$ & $50 / 680$ & $56 / 788$ & -
\end{tabular}




\section{Acknowledgements}

Supported by the grant 201/07/0294 of the Grant Agency of the Czech Republic and by the Research Project MSM6198910027 of the Czech Ministry of Education.

\section{References}

[1] L. Baillet, T. Sassi. Simulations numériques de différentes méthodes d'éments finis pour les problémes contact avec frottement. C. R. Acad. Sci, Paris, Ser. IIB, 331 (2003),789-796.

[2] G. Bayada, J. Sabil, T. Sassi. Algorithme de décomposition de domaine pour un probléme de Signorini sans frottement. C. R. Acad. Sci. Paris, Ser. I335 (2002), 381-386.

[3] G. Bayada, J. Sabil, T. Sassi. A Neumann-Neumann domain decomposition algorithm for the Signorini problem. Appl. Math. Letters, 17 (2004), 1153-1159.

[4] P. E. Bjorstad, O. B. Widlund. Iterative methods for the solution of elliptic problems on regions partitioned into substructures. SIAM J. Numerical Analysis, 23 (1986), No. 6, 10971120.

[5] P. W. Christensen, A. Klarbring, J. S. Pang, N. Strömberg. Formulation and comparison of algorithms for frictional contact problems. Internat. J. Numer. Methods Engrg., 42 (1998), No. 1, 145-173.

[6] Z. Dostál, J. Schöberl. Minimizing quadratic functions over non-negative cone with the rate of convergence and finite termination. Comput. Optim. Appl., 30 (2005), No. 1, 23-44.

[7] C. Eck, B. Wohlmuth. Convergence of a Contact-Neumann iteration for the solution of twobody contact problems. Mathematical Models and Methods in Applied Sciences, 13 (2003), No. 8, 1103-1118.

[8] R. Glowinski, J. L. Lions, R. Trémoliére. Numerical analysis of variational inequalities. Studies in Mathematics and its Applications, Volume VIII, North-Holland, Amsterdam, 1981.

[9] G. H. Golub, C. F. Van Loan. Matrix computation. The Johns Hopkins University Press, Baltimore, 1996.

[10] J. Haslinger, Z. Dostál, R. Kučera. On a splitting type algorithm for the numerical realization of contact problems with Coulomb friction. Comput. Methods Appl. Mech. Engrg., 191 (2002), No. 21-22, 2261-2281.

[11] J. Haslinger, I. Hlaváček, J. Nečas. Numerical methods for unilateral problems in solid mechanics. Handbook of Numerical Analysis, Volume IV, Part 2, North Holland, Amsterdam, 1996. 
[12] M. A. Ipopa. Algorithmes de Décomposition de Domaine pour les problémes de Contact: Convergence et simulations numériques. Thesis, Universit de Caen, 2008.

[13] N. Kikuchi, J. T. Oden. Contact problems in elasticity: A study of variational inequalities and finite element methods. SIAM, Philadelphia, 1988.

[14] R. Kornhuber, R. Krause. Adaptive multigrid methods for Signorini's problem in linear elasticity. Comput. Vis. Sci., 4 (2001), No. 1, 9-20.,

[15] R. Krause, B. Wohlmuth. A Dirichlet-Neumann type algorithm for contact problems with friction. Comput. Vis. Sci., 5 (2002), No. 3, 139-148.

[16] P. Le Tallec. Domain decomposition methods in computational mechanics. Comput. Mech. Adv., 1 (1994), No. 2, 121-220.

[17] J. Sabil. Modélisation et méthodes de décomposition de domaine pour des problémes de contact. Thesis, INSA de Lyon, 2004. 